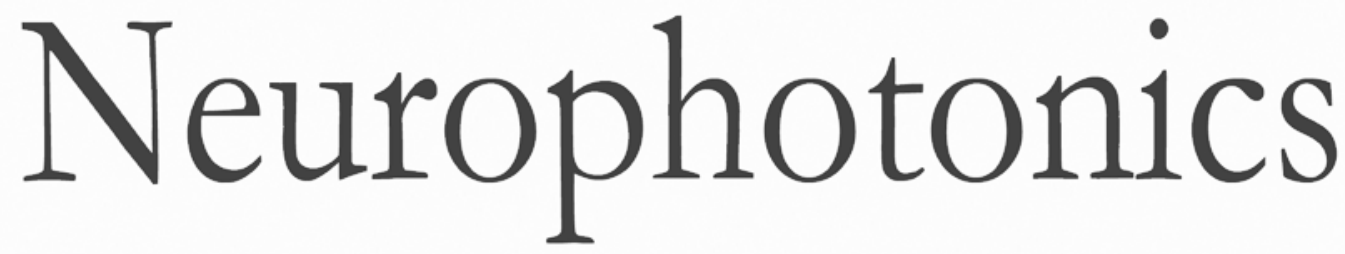

\title{
Genetically expressed voltage sensor ArcLight for imaging large scale cortical activity in the anesthetized and awake mouse
}

Peter Y. Borden

Alex D. Ortiz

Christian Waiblinger

Audrey J. Sederberg

Arthur E. Morrissette

Craig R. Forest

Dieter Jaeger

Garrett B. Stanley 


\title{
Genetically expressed voltage sensor ArcLight for imaging large scale cortical activity in the anesthetized and awake mouse
}

\author{
Peter Y. Borden, ${ }^{\text {a }}$ Alex D. Ortiz, ${ }^{a}$ Christian Waiblinger, ${ }^{a}$ Audrey J. Sederberg, ${ }^{a}$ Arthur E. Morrissette, ${ }^{b}$ \\ Craig R. Forest, ${ }^{c}$ Dieter Jaeger, ${ }^{b}$ and Garrett B. Stanley ${ }^{\mathrm{a}, *}$ \\ ${ }^{a}$ Georgia Institute of Technology and Emory University, Wallace H. Coulter Department of Biomedical Engineering, Atlanta, Georgia, United States \\ ${ }^{b}$ Emory University, Neuroscience Program, Department of Biology, Atlanta, Georgia, United States \\ ${ }^{c}$ Georgia Institute of Technology and Emory University, School of Mechanical Engineering, Atlanta, Georgia, United States
}

\begin{abstract}
With the recent breakthrough in genetically expressed voltage indicators (GEVIs), there has been a tremendous demand to determine the capabilities of these sensors in vivo. Novel voltage sensitive fluorescent proteins allow for direct measurement of neuron membrane potential changes through changes in fluorescence. Here, we utilized ArcLight, a recently developed GEVI, and examined the functional characteristics in the widely used mouse somatosensory whisker pathway. We measured the resulting evoked fluorescence using a widefield microscope and a CCD camera at $200 \mathrm{~Hz}$, which enabled voltage recordings over the entire cortical region with high temporal resolution. We found that ArcLight produced a fluorescent response in the S1 barrel cortex during sensory stimulation at single whisker resolution. During wide-field cortical imaging, we encountered substantial hemodynamic noise that required additional post hoc processing through noise subtraction techniques. Over a period of 28 days, we found clear and consistent ArcLight fluorescence responses to a simple sensory input. Finally, we demonstrated the use of ArcLight to resolve cortical S1 sensory responses in the awake mouse. Taken together, our results demonstrate the feasibility of ArcLight as a measurement tool for mesoscopic, chronic imaging. ๑ 2017 Society of Photo-Optical Instrumentation Engineers (SPIE) [DOI: 10.1117/1.NPh.4.3.031212]
\end{abstract}

Keywords: voltage sensor; genetically expressed voltage indicator; cortex; in vivo; imaging; ArcLight.

Paper 17006SSR received Jan. 18, 2017; accepted for publication Apr. 10, 2017; published online May 4, 2017; corrected Jul. 3 , 2017.

\section{Introduction}

With the recent breakthrough in genetically expressed voltage indicators (GEVIs), ${ }^{1}$ there has been a tremendous demand to quantify the capabilities of these sensors in vivo. Novel voltage sensitive fluorescent proteins allow for direct measurement of membrane potential changes through changes in fluorescence. These GEVIs, including ArcLight, ${ }^{2}$ VSFP $2.3,{ }^{3}$ Butterfly 1.2, ${ }^{4}$ Quasar $1,{ }^{5}$ and mNeon-Ace, ${ }^{6}$ have shown incredible promise to record neural responses. ${ }^{7,8}$ However, these approaches have not yet been widely applied in scientific studies in the mammalian nervous system (for review Refs. 8 and 9). Although recent calcium probes have greatly increased the understanding of complex neural systems, they still offer only moderate temporal resolution (50 to $100 \mathrm{~ms})^{10}$ and report only on byproducts of suprathreshold neural spiking activity through calcium responses. Additionally, many studies try to deconvolve the calcium signal to glean information about ongoing membrane potential with mixed success (for review Ref. 11). In contrast, voltage sensors allow for fast temporal information (i.e., milliseconds) and have the potential to report even subthreshold information.

We present an investigation into the functional capabilities of ArcLight, one of the sensors available as an in vivo probe of wide-field cortical signals. ArcLight, ${ }^{1}$ a modified GFP protein, has fast temporal resolution $(\sim 10 \mathrm{~ms})$ with relatively large changes in fluorescence in response to membrane fluctuations.

*Address all correspondence to: Garrett B. Stanley, E-mail: garrett.stanley@ bme.gatech.edu
Although several investigators have demonstrated the capabilities of ArcLight, most of these studies have reported responses through in vitro models, ${ }^{12,13}$ Drosophila, ${ }^{2,14-17}$ or in the mouse olfactory system. ${ }^{2,18}$ Other sensors, including VSFP 2.3 (Ref. 3) and Butterfly 1.2, ${ }^{19}$ have been previously shown to be successful for in vivo cortical sensory recordings (for review Ref. 8). In this work, however, we are the first, to our knowledge, to demonstrate the use of ArcLight in cortical structures in the awake and anesthetized mammalian brain.

Here, we demonstrate that ArcLight produces a robust and reliable sensory evoked fluorescent response in the $\mathrm{S} 1$ barrel cortex to sensory stimulation. We found that in the S1 barrel cortex the spectral overlap with the hemodynamic activity was substantial in its raw form and required long imaging experiments and trial averaging to reduce noise. In order to address this large hemodynamic signal, we subtracted a scaled OffROI signal to remove ongoing noise. Although this method dramatically removes the hemodynamic response, there are several assumptions and concerns that limit the widespread use of this technique. Using this post hoc subtraction method, we found that the evoked response matched the fast temporal dynamics of other voltage indicators including voltage sensitive dye (VSD) RH1691, ${ }^{20-23}$ VSFP $2.3,{ }^{23}$ and Butterfly $1.2 .{ }^{4}$ ArcLight showed clear stimulus-evoked fluorescence for stimuli with frequency content up to $20 \mathrm{~Hz}$ with high fidelity. Using paired local field potential (LFP) recordings, we determined a high correlation between the average LFP and ArcLight signals in response to

$2329-423 X / 2017 / \$ 25.00$ (C) 2017 SPIE 
sensory stimuli; however, on a single trial the two signals showed weak correlation. Finally, we were able to resolve sensory evoked fluorescence in awake mice. Based on these results, we conclude that ArcLight has a capacity to measure chronic in vivo cortical responses. ArcLight would be suited for in vivo experiments where a single fluorophore sensor is desired, in particular experiments that require long bouts of continuous imaging.

\section{Methods}

All procedures were approved by the Georgia Institute of Technology Institutional Animal Care and Use Committee and followed guidelines established by the National Institutes of Health.

\subsection{Virus Delivery}

At least four weeks prior to experimentation, six-week-old female mice (C57BL/6, Jackson Laboratories) were anesthetized using isoflurane, $3 \%$ to $5 \%$ in a small induction chamber, and maintained at $1 \%$ to $3 \%$ isoflurane. Following anesthetization, 1 to 2 small craniotomies were created over the barrel field of the primary somatosensory cortex (S1) according to stereotaxic measurements taken from the bregma $(3.5 \mathrm{~mm} \times 1.5 \mathrm{~mm}$, and $2.5 \mathrm{~mm} \times 3 \mathrm{~mm})$. The virus was loaded into a Hamilton syringe (701-N). A custom $\sim 35-\mu \mathrm{m}$ pulled borosilicate glass pipette was filled with a silicone gel and secured onto the tip of the Hamilton syringe to increase taper and to reduce damage to the cortex caused during the injection. The injection pipette was initially lowered to the target depth below the pia surface $(500 \mu \mathrm{m})$ using a $10-\mu \mathrm{m}$ resolution stereotaxic arm (Kopf, Ltd.). Following a 1-min delay to allow for tissue relaxation, each animal was injected with $1 \mu \mathrm{L}$ of adeno-associated virus (AAV)1-hsyn1-ArcLight-D-WPRESV40 (UPenn Viral Vector Core, AV-1-36857P) at a flow rate of $0.1-\mu \mathrm{L} / \min (0.5 \mu \mathrm{L}$ each for two injections). After injection, the pipette remained in place for an additional 5 min before slowly being removed from the brain. The craniotomies were then filled with bone wax or left to close naturally. In all cases, the skull was sealed by clamping the skin using wound clips. During the injection, mice were kept warm using a water heating system to maintain body temperature. Throughout the experiment, sterile techniques were used to keep the injection area clean and free from infection. Additionally, no antibiotics were given to prevent infection. All mice survived this minor procedure.

\subsection{Headplate and Surgical Preparation}

After at least four weeks postinjection, we secured a metal headplate to the skull for fixation in order to reduce vibration and allow head fixation during imaging experiments. The custom metal headplate (titanium) formed a ring (inner radius $5 \mathrm{~mm}$ ) around the entire cortex and contained flared $\mathrm{v}$-shape projections $(\sim 10 \mathrm{~mm})$ for attachment to a custom vice to reduce vibration. Mice were initially anesthetized using isoflurane (3\% to $5 \%$ ) and then placed on a heated platform (FHC, Inc.) with a stereotaxic nose cone to maintain anesthesia. A large incision was made over the skull. The connective tissue and muscles surrounding the skull were removed using a fine scalpel blade (Henry Schein \#10). A headplate was attached using a three stage dental acrylic, Metabond (Parkell, Inc.). The Metabond was chilled using ice, slowly applied to the surface of the skull, and allowed to cure for 5 to $10 \mathrm{~min}$. After securing the headplate, the skull was left either exposed or was lightly thinned using a dental drill and covered with a thin layer of clear adhesive (LockTight 401, ULine, Inc.). We found that the Metabond dental acrylic alone was able to firmly adhere to the animal's skull and could not be removed without destroying the adhered bone. During preparation for histological validation, the headplate could not be separated from the attached skull and the brain was extracted by removing the lower jaw. The final headplate and dental acrylic structure additionally created a well for saline that helped maintain skull transparency for imaging during the intact skull preparation. The headplate was then transferred to a flexible arm to align the camera for imaging of the cortex. The nose cone was realigned to allow for continuous delivery of isoflurane while having access to the whiskers. After surgery, the isoflurane levels were dropped to $\sim 1 \%$ for all imaging and electrophysiological experiments. The animal's heart rate, respiratory rate, blood oxygenation, and toe-pinch responses were constantly measured for anesthesia depth. Isoflurane levels were adjusted to maintain a constant level of light anesthetization, monitored by heart rate, respiration rate, and functional cortical response.

\subsection{Whisker Stimulator}

All single whiskers were stimulated by a galvanometer system (Cambridge Technologies) to yield high-fidelity sensory stimuli. The galvanometer stimulator has a $15-\mathrm{mm}$ extension to target single whiskers. The galvanometer system was controlled using a custom developed hardware/software system (MATLAB Realtime Simulink, Mathworks). The real-time system controls the stimulus using two computers, a target and a host. The target computer ran a proprietary Linux kernel that was controlled by the host computer. The entire system was updated at a 1-kHz sampling rate, with a custom developed algorithm to output voltage commands using an analog output card (National Instruments). The galvanometer system was positioned $\sim 10 \mathrm{~mm}$ from the mouse whisker pad and delivered deflections on the single whisker in the rostral-caudal plane. Unless otherwise noted, we used a simple exponential sawtooth (rise and fall time $=8 \mathrm{~ms}$ ) for punctate whisker deflections. ${ }^{24}$ The reported waveform stimulus velocity was determined as the average velocity during the waveform (1200 deg/s). All stimulus waveforms were delivered in a pseudorandom order with at least $4 \mathrm{~s}$ between trials to reduce potential confounds. Due to the fast rising edge of the sawtooth, all latencies were defined relative to stimulus onset.

\subsection{Cortical ArcLight and Intrinsic Imaging}

ArcLight transfected mice were imaged through either intact or thinned skull using a wide-field fluorescence imaging system to measure cortical spatial activity (MiCam02HR Scimedia, Ltd.). During all imaging experiments, isoflurane anesthesia levels were lowered to $\sim 1 \%$. The headplate was used as a saline well to keep the bone surface wet during imaging, which dramatically increases transparency of the mouse's skull. Some animals were chronically imaged through either intact or thinned skull covered with a glass coverslip and/or cyanoacrylate glue. The cortex was imaged using a $184 \times 123$ pixel CCD camera (Scimedia MiCam2 HR Camera) at $200 \mathrm{~Hz}$. In all experiments, we had a field of view of $4 \times 3 \mathrm{~mm}$ with a total of a 1.6 magnification (48 pixels $/ \mathrm{mm}$ ). The particular optical system used in this 
work has an optical resolution of $2.25 \mu \mathrm{m}$ (numerical aperture $=$ 0.141 , optical resolution $=0.61 \lambda / \mathrm{NA}$ ). The camera in combination with the optics had a spatial resolution of $\sim 20 \mu \mathrm{m}$ per pixel; however, this resolution does not consider the scattering of the light in the tissue. During experimental imaging, the illumination excitation light was left continuously on. The entire cortical area was illuminated at $465 \mathrm{~nm}$ with a $400-\mathrm{mW} / \mathrm{cm}^{2}$ LED system (Scimedia, Ltd.) to excite the ArcLight fluorophore. The excitation light was further filtered (cutoff: 472/30-nm bandpass filter, Semrock, Inc.) and projected onto the cortical surface using a dichroic mirror (cutoff: $495 \mathrm{~nm}$, Semrock, Inc.). Collected light was filtered with a bandpass emission filter between wavelengths of 520/35 nm (Semrock, Inc.). The imaging system was focused at $\sim 300 \mu \mathrm{m}$ below the cortical surface to target cortical layer $2 / 3$. The procedures for mapping and recording sensory responses in the barrel cortex with the ArcLight voltage sensor are outlined below. For intrinsic imaging of the hemodynamic response, the cortical surface was illuminated by a $625-\mathrm{nm}$ red LED (ThorLabs) and imaged with the same camera system as above, at a temporal resolution of $10 \mathrm{~Hz}$. During intrinsic imaging, no emission filters were used. In order to evoke a cortical intrinsic response, the whisker was repetitively stimulated at $10 \mathrm{~Hz}$ for $6 \mathrm{~s}$.

\subsection{Recording ArcLight Fluorescent Sensory Responses in the S1 Barrel Cortex}

The mouse's whisker system was first mapped by imaging the rapid response to a high-velocity (1200 deg /s) sensory stimulus to at least three whiskers. We used three criteria to localize and isolate the barrel cortex: stereotaxic localization, relative evoked temporal response, and topographic mapping of cortical activation. All imaging experiments were centered on standard stereotaxic location of $\mathrm{S} 1$ ( $\sim 3 \mathrm{~mm}$ lateral, 0.5 to $1.5 \mathrm{~mm}$ caudal from bregma). The resulting whisker responses were averaged over 20 trials. The response was determined to likely be from the barrel cortex if the average evoked fluorescence at the onset of the evoked response (20 to $25 \mathrm{~ms}$ after stimulation) was spatially limited to $\sim$ a $250 \mu \mathrm{m} \times 250 \mu \mathrm{m}$ area. Additionally, another criterion for functionally identifying S1 barrel cortex was through topographic mapping - if the center of mass of activation across whiskers moved consistently with the post hoc histologically identified barrels, activity was attributed to the barrel cortex. In some cases, S2 activation was detected in response to whisker deflection and was rejected based on an extreme lateral response ( $\sim 3.5$ to $4 \mathrm{~mm}$ from midline) and lack of a clear topographic representation of the whisker barrels. Once the barrel field was appropriately mapped, we selected a single whisker to be deflected for the entire experiment.

\subsection{Simultaneous Blood Oxygenation Measurements}

During experiments where the combination of blood oxygenation and blood flow was simultaneously captured, a small LED sensor was attached to the rodent's hindpaw. The specific sensor (Easy Pulse sensor v1.1, Embedded Lab) was modified to have frequency filtering within the typical rodent heart rate (analog low-pass filter cutoff: $15 \mathrm{~Hz}$ ). The recorded value measures the changes in the absorption of infrared light $(\sim 940 \mathrm{~nm})$ to measure changes in blood oxygenation (and blood flow) over time. The reported values of the heart rate generated from the custom oxygenation sensor were cross validated with blood flow recordings of the commercially available physiological suite (Kent Scientific). Paired blood flow recordings and imaging was achieved using the simultaneous analog inputs in to the camera system at $4 \mathrm{kHz}$ (MiCam02HR, Scimedia, Ltd.).

\subsection{Simultaneous Local Field Potential Recordings and Analysis}

In a subset of experiments, we simultaneously recorded the LFP along with the ArcLight imaging, using a similar prep as described above (see Sec. 2.4). After mapping the mouse cortical barrels, we removed a small portion of the bone over the selected barrel $(\sim 1.5 \mathrm{~mm} \times 1.5 \mathrm{~mm}$ area $)$ to have access to the underlying cortical surface. We lowered a low impedance tungsten electrode $(<500 \mathrm{k} \Omega$, FHC Inc.) using a micromanipulator (Luigs \& Neumann) to $300 \mu \mathrm{m}$ below the cortical surface to approximately layer $2 / 3$. We identified the principle whisker through repetitive manual stimulation of different single whiskers. Once we localized the principle whisker, we attached the whisker stimulator and applied the sensory stimulus (above). We recorded electrophysiological data using a 128-channel cerebus system (Blackrock Microsystem LLC.) continuously sampled at $2 \mathrm{kHz}$. All LFP signals were notch filtered at $60 \mathrm{~Hz}$ to remove any electrical noise. Furthermore, we normalized LFP signals on a trial-by-trial basis by subtracting the average $200 \mathrm{~ms}$ prestimulus activity. In all cases, a zero-phase filter approach was utilized using custom MATLAB (Mathworks) scripts.

\subsection{Chronic Multiday Imaging under Anesthesia}

Three mice were first injected with the AAV construct and were outfitted with a custom developed headplate device to maintain stable recordings (see above). In order to increase the fluorescence recorded, the mouse's skull was thinned to $\sim 25 \%$ of the original thickness (or until transparent) using a surgical drill over the injected region (roughly $3 \mathrm{~mm} \times 3 \mathrm{~mm}$ ). After thinning, the mouse's skull was sealed using clear adhesive (Loctite 401 Adhesive, Uline). During skull thinning, the ArcLight fluorescent responses were briefly mapped to identify and localize the barrel cortex. After implantation, mice were left to recover for at least 1 week before imaging again. Day 1 corresponds to the first imaging experiment after 1 week of recovery post headplate implantation. The same mouse whisker (A1) was imaged over the course of 28 days, specifically on days 1, 3, 5, 7, 14, 21, 28. Isoflurane anesthesia was held to similar levels across imaging experiments by maintaining heart rate between 500 and $600 \mathrm{bpm}$, monitored from the animal's paw (Kent Scientific, $\sim 1 \%$ isoflurane). During imaging the mouse cortical responses were mapped with at least two whiskers to identify the correct region and presented with a velocity stimulus (described above). The entire imaging experiment lasted $\sim 1$ to $2 \mathrm{~h}$ each day. After imaging, the cortical surface was covered with a silicone plug (Kwik-Cast, World Precision Instruments LLC) to prevent photobleaching of the fluorophore between experiments. Mice were imaged only during the specific time points listed above.

\subsection{Awake Imaging}

At least four weeks after ArcLight viral injection, mice were anesthetized under isoflurane and were headplated using the above stated protocol. Over the course of 3 days preceding the first imaging experiment, mice were routinely handled to gain familiarity with the imaging system and immobilization 
device. During this acclimatization period, mice were increasingly head fixed for longer periods of time, for 15, 30, and 45 min, respectively. During stimulation of the whisker, mice were prevented from interacting with the whisker stimulator by obstructing the path from the paws to the whisker. Mice were rewarded with sweetened milk (Nestle, Ltd.) throughout imaging, which greatly helped to reduce animal frustration. After 3 days of handling and acclimating, mice appeared to be calm while the head was immobilized in the headplate restraint system. During passive stimulation of the whiskers, the mice often actively moved their whiskers. Therefore, the galvanometer was placed $5 \mathrm{~mm}$ from the face to prevent the whisker from slipping out of the manipulator; however, the amplitude of the deflection was adjusted to maintain a consistent velocity stimulation $(1200 \mathrm{deg} / \mathrm{s})$ as presented in the anesthetized case (see above).

\subsection{Histology}

Histological samples were prepared by perfusing the animal transcardially with phosphate buffered saline (PBS) followed by $4 \%$ paraformaldehyde. Brains were postfixed overnight in $4 \%$ paraformaldehyde then transferred to PBS before sectioning. Thick sections were cut using a vibratome (100 $\mu \mathrm{m}$, Leica) and either directly mounted or saved for staining. In some cases, we cryosectioned the postfixated brains to achieve thinner sections $(20 \mu \mathrm{m})$ for better imaging. Before sectioning, samples were submerged in $30 \%$ sucrose in PBS post fixation until saturated with sucrose (causing the tissue to sink). The tissue was then snap frozen and embedded in optimal cutting temperature compound (Tedpella, Inc.). Thin sections were cut on a cryotome $(20 \mu \mathrm{m})$. ArcLight was stained against using a Rabbit anti-GFP polyclonal antibody (Abcam) and Alexa 488 secondary (Life Technologies, Inc.). After staining, the sections were then counterstained with Nissl (Neurotrace 640 Life Technologies, Inc.) to isolate neurons. ArcLight was imaged using the 405-nm laser on an NLO 710 confocal microscope (Zeiss) and processed using Zen software (Zeiss).

\subsection{Voltage Imaging Data Analysis}

In this section, we have limited our description of the analytical methods used to the processing of the raw fluorescence signal. For specific description of the methods for each figure shown, see the corresponding results Secs. 3.2 and 3.3. All data analyses for ArcLight imaging were accomplished using custom written image-analysis software (MATLAB 2015a, Mathworks, Inc.). A general outline of the image analysis is shown in Fig. 3.

Raw images were loaded and converted from the proprietary file format of the imaging system using custom scripts. Due to the natural decay of the fluorescent signal caused by photobleaching, each trial was first normalized to a baseline and reported as a percent change in fluorescent activity $\left(\% \Delta F / F_{0}\right)$. The $\Delta F / F_{0}$ measurement was calculated by subtracting and dividing each trial's fluorescence $F(x, y, t)$ by the frame preceding the stimulus delivery

$$
\frac{\Delta F}{F_{0}}=\frac{F-F_{0}}{F_{0}},
$$

where $F_{0}(x, y)$ is the frame of stimulus delivery $\left(F_{0}=F\right.$ at $t=0)$. A single region of interest (ROI) was identified using the largest $9 \times 9$ pixel $(\sim 150 \times \sim 150 \mu \mathrm{m})$ area response at $25 \mathrm{~ms}$ post $1200 \mathrm{deg} / \mathrm{s}$ stimulus onset.

After normalization to a $\Delta F / F_{0}$ measurement, the signal still contained a large component of hemodynamic noise [see Fig. 3(b)]. The observed noise was determined to be centered around 7 to $10 \mathrm{~Hz}$, which corresponded with the animals' ongoing heartbeat [see Fig. 3(b), Appendix A1]. This hemodynamic noise was removed using a highly correlated ROI (Off-ROI). This Off-ROI was defined as the $9 \times 9$ average pixel region $(\sim 150 \times \sim 150 \mu \mathrm{m}$ area $)$ at least 48 pixels $(\sim 1 \mathrm{~mm})$ away from the ROI with the highest average correlation of fluorescence during the first nonstimulus trial (see Appendix A2). The Off-ROI region was fixed during all subsequent trials. The separation of $1 \mathrm{~mm}$ typically results in a background measurement that is highly correlated with the ROI while avoiding the evoked response. We found that this distance did not cause changes in the evoked mean response [see Fig. 3(f) and corresponding results Sec. 3.2 and Appendix A3], while allowing for subtraction of ongoing hemodynamic fluctuations.

In each trial, the activity in the Off-ROI $200 \mathrm{~ms}$ preceding stimulus delivery was projected onto the ROI using a linear regression model [Fig. 3(c)], which was then removed from the activity within the ROI to produce the final time series data that were used for all calculations (see Fig. 3 for more details). Due to the fluorophore, ${ }^{1}$ positive changes in membrane potential correspond to a decrease in ArcLight fluorescent activity. Therefore, all traces here have been inverted to show a decrease in fluorescence as an increase in magnitude for aesthetic purposes.

\subsection{Statistics}

In all cases, we first determined if the specific data sets were normally distributed using the Lilliefors test for normality. ${ }^{25}$ If the data were normal, we used the appropriate (paired or unpaired) $t$-test for statistical difference. If the population was determined to have nonnormal distributions, we conducted nonparametric Wilcoxon signed-rank tests to determine statistical significance. All tests were conducted using the MATLAB Statistics Toolbox (Mathworks, Inc.).

\section{Results}

\subsection{Experimental Setup and Histological Validation of Genetic Expression in Barrel Cortex}

We validated the location of expression of the ArcLight injections in the S1 barrel cortex through postexperiment fixation and histological analysis of transfected animals. We localized the GEVI ArcLight in the mouse barrel cortex by injecting $1 \mu \mathrm{L}$ of AAV1-hsyn1-ArcLight-D-WPRE-SV40 (UPenn Viral Vector Core, AV-1-36857P) using stereotaxic coordinates and a microinjector system (see Sec. 2.3). Similar to other published work, we found that under the human synapsin promotor (hsyn1), ArcLight expressed predominantly in layers $2 / 3$ and 5 of the mouse cortex ${ }^{26}$ [Figs. 1(b)-1(c)]. Based on the limitations of blue light penetration to the superficial cortical layers, our recorded fluorescence signals are a combination of layer $2 / 3$ somatic, axonal, and dendritic information along with layer 5 apical dendrites. Under further magnification, ArcLight appeared to express across the cellular membrane [Fig. 1(d)], suggesting that the wide-field response is a combination of all membrane related neural responses from the expressed areas 
(a)

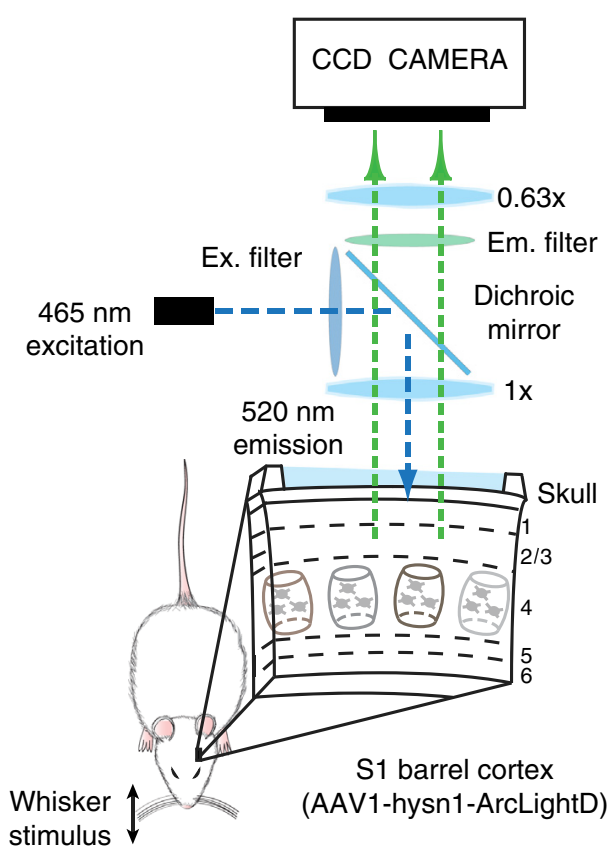

(b)

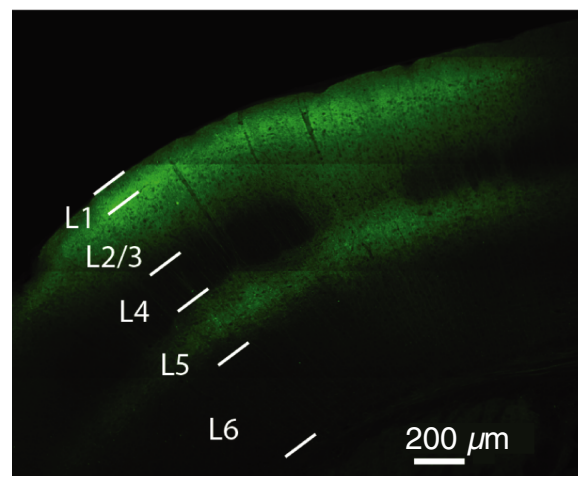

(c)

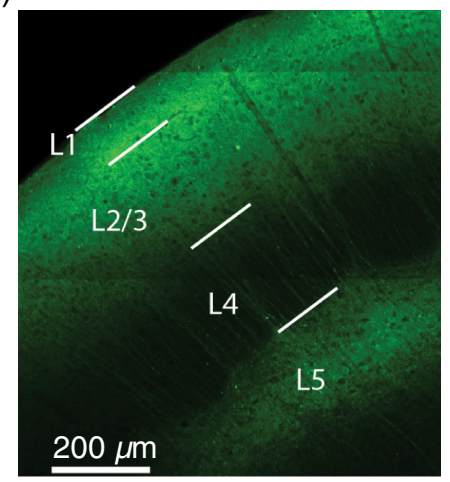

(d) Anti-GFP stain

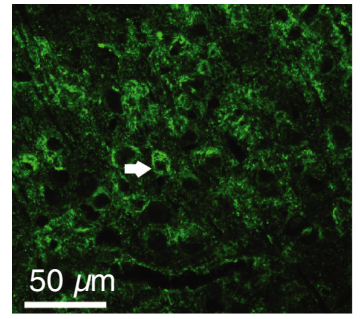

(e)

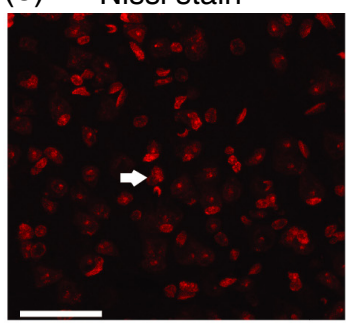

(f) Merged

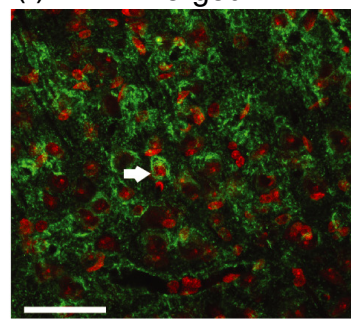

Fig. 1 Experimental setup and histological validation of ArcLight expression. (a) The experimental setup for ArcLight imaging. (b) Confocal image of the characteristic spread of ArcLight in the S1 barrel cortex (see Sec. 2). Fluorescence (green) from ArcLight excited with 465-nm LED. Layers based on characteristic depths are outlined in white, cross validated with Nissl stain. (c) Confocal image of ArcLight expression. The ArcLight expression can be clearly seen across layer 2/3 and layer 5. (d) Confocal image of ArcLight expression in cortical region cryosectioned and stained using an anti-GFP polyclonal antibody. Fluorescence is clearly expressing in the neural membranes. An example cell is highlighted with the white arrow. (e) Same section as (d) stained with Nissl (red) for identification of neural cell bodies. (f) Merged image from (d) and (e) shows fluorescent expression in membranes surrounding Nissl (red) stained neural somas. Expression appears to be targeted to somatic, dendritic, and axonal neural membranes.

(i.e., soma, dendrite, axon). ArcLight has been shown in previous work to highly express in the cellular membrane. ${ }^{1,27}$ We confirmed the expression of ArcLight to the neural membrane, through anti-GFP [Fig. 1(d)] and Nissl [Fig. 1(e)] staining, the combination of which is shown in Fig. 1(f). ArcLight is derived from the GFP molecule, ${ }^{2}$ and therefore, was counterstained with polyclonal anti-GFP molecules to improve signal to noise over background fluorescence. These observations of ArcLight neuronal membrane expression are highly consistent with recent work in the olfactory bulb under similar conditions with the hsyn 1 promoter. ${ }^{18}$ The histology highlights the ability of ArcLight, under hsyn 1 promoter, to genetically target all neural membranes, which offers higher selectivity than traditional VSDs that bind to all cellular membranes (neuronal and glial).

\subsection{ArcLight Response to Single Whisker Deflections}

We measured the spatio-temporal ArcLight fluorescence in the cortex using a fluorescence microscope and a CCD camera system [imaged at $200 \mathrm{~Hz}$, for setup see Fig. 1(a)]. We first applied our sensory stimuli to a single mouse whisker using a customized actuator (see Sec. 2) and recorded the evoked fluorescence response in the primary somatosensory cortex (S1) (Fig. 2). Specifically, we presented a strong (1200 deg/s) stimulus to a single whisker and recorded the evoked fluorescent cortical response [Fig. 2(a)]. Stimulus features such as these have been widely used across a range of laboratories including our own ${ }^{24,28}$ inspired by high-velocity transients of whisker motion observed in active sensing. ${ }^{29-31}$

In response to these single whisker pulsatile deflections, we observed a change in cortical fluorescence consistent with the reported topography of S1 barrel cortex. Each imaging experiment consisted of 50 to 100 trials, which were subsequently averaged, and postprocessed using the stated methods (Sec. 2.11). Unless otherwise noted, we utilized an Off-ROI subtraction method to remove the hemodynamic signal on a pixel by pixel basis across the entire image. For every experiment, an Off-ROI was selected, scaled, and subtracted from the ROI to reduce ongoing hemodynamic noise (see Sec. 2.11 and Fig. 3 for more details of this procedure). Note that the Off-ROI subtraction method produced similar results to the raw averaged signal (data not shown). Similar to published wide-field VSD imaging, ${ }^{28,32-35}$ the recorded voltage response initially started in a small region approximately the size of a single mouse barrel and rapidly increased to a much larger area encompassing much of the barrel cortex [Fig. 2(a), at $\sim 35 \mathrm{~ms}$ poststimulus]. The fluorescence then decayed over the course of $\sim 600 \mathrm{~ms}$, until returning to baseline activity. We calculated the total area of activation by normalizing each dataset as a percent change 
(a)

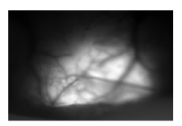

$30 \mathrm{~ms}$ $\operatorname{lng} 20$

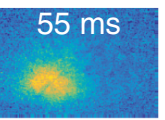

Single experiment mean response

(102 trials)

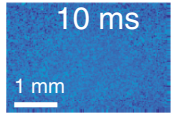

$35 \mathrm{~ms}$
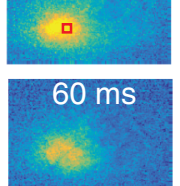
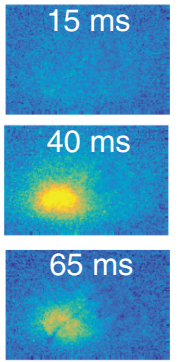
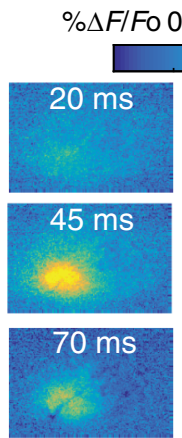

$-0.2-0.4$

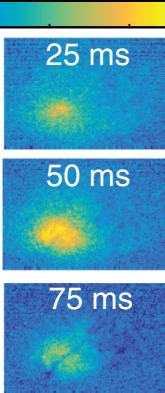

(b) Single experiment mean response ( \pm SEM, 102 trials)

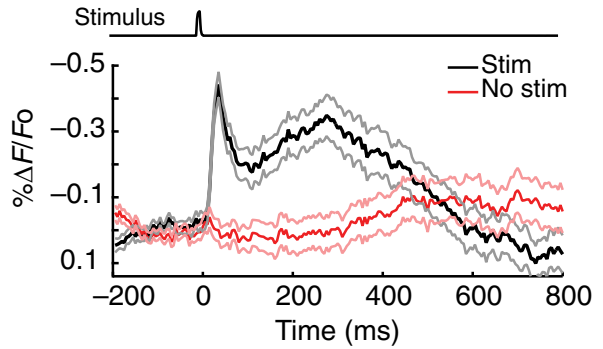

(d)

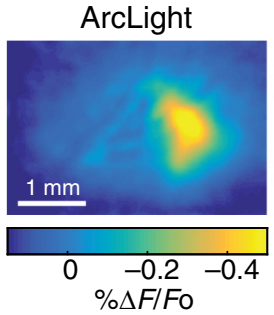

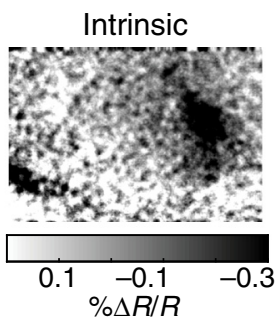

(c)

Grand average response ( \pm SEM, $n=31$ )

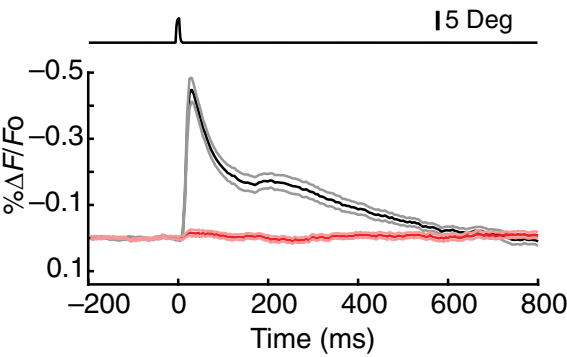

(e)

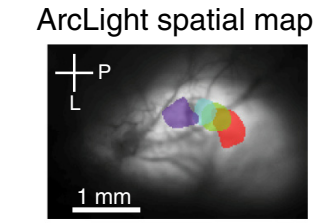

$\begin{array}{llll}\mathrm{D} 1 & \mathrm{C} 1 & \mathrm{~B} 1 & \mathrm{~A} 1\end{array}$

Fig. 2 ArcLight spatial and temporal S1 cortical response to punctate deflection. (a) Single session ArcLight fluorescent response to single whisker deflection. Top numbers in each frame represents the time poststimulus, captured at $200 \mathrm{~Hz}$. Each frame is normalized to the frame at stimulus delivery and averaged over 102 trials of stimulus presentation. All data shown in this figure have also been postprocessed using the Off-ROI subtraction method shown in Figs. 3 and Fig. 11 (see Sec. 2.11). The signal starts at time 20-ms poststimulus and grows to activate a larger region of the barrel field and slowly dissipates back to baseline fluorescence. (b) Mean single session temporal response from a single $150 \times$ $150 \mu \mathrm{m}$ region [red square $35 \mathrm{~ms}$ post in (a)] within the spatial activity in (a) (102 trials) \pm SEM. Top trace represents galvanometer input to the whisker system. (c) Grand average temporal response within the peak $150 \times 150 \mu \mathrm{m}$ region ( $n=31$ experiments, across 8 animals). (d) Spatial comparison between ArcLight response (left, 102 trials) and Intrinsic (right, 10 trials) response in the same animal reveals similar localization of activation to S1 barrel cortex. Intrinsic response captured at $10 \mathrm{~Hz}$ with $625-\mathrm{nm}$ excitation. ArcLight spatial image represents the mean 40- to 100-ms response to an 11-deg ramp and hold deflection. Intrinsic spatial image represents the mean 0.8- to 2-s response to during a 6-s $10-\mathrm{Hz} 1500 \mathrm{deg} / \mathrm{s}$ pulsatile stimulus. (e) Evoked activity map generated by stimulating four whiskers independently (D1, C1, B1, A1). Overlay represents a $50 \%$ contour of the fluorescent sensory signal.

over each trial's baseline activity $\left(\% \Delta F / F_{0}\right.$, see Sec. 2.11$)$, and spatially smoothing the images with a small $100 \times 100 \mu \mathrm{m}$ Gaussian filter (similar to Ref. 28). The total area of activation was calculated as the cortical area corresponding to the $50 \%$ contour of the mean peak response between 25 and $35 \mathrm{~ms}$ poststimulus (see Sec. 2.11). We measured the initial spatial response to be on average $1.92 \pm 0.879$ [standard deviation (SD)] $10^{5} \mu \mathrm{m}^{2}(N=31$ experiments, 7 animals). This initial activation corresponds to a region of $\sim 425 \times 425 \mu \mathrm{m}$, or 1 to 2 cortical barrels. ${ }^{36,37}$ These results correlate well with intracellular recordings from layer $2 / 3$ cortical neurons that show that surrounding barrels receive subthreshold sensory input from a single whisker. ${ }^{38}$
In order to determine if the area of expression caused by the viral injection changed the observed evoked spatial response, we compared the evoked spatial response across different mice with different patterns of ArcLight expression. Using a noninjected mouse, we determined the overall level of baseline autofluorescence with our imaging system. We developed a threshold (two times the baseline average autofluorescence from the noninjected mouse) to approximate the area expressing the ArcLight protein. We found that our cortical injections produced expression across $\sim 50 \%( \pm 19.4 \% \mathrm{SD})$ of the recorded $4 \mathrm{~mm} \times 3 \mathrm{~mm}$ field of view. We found no correlation between the expressed area and the evoked spatial response $\left(R^{2}=0.075\right)$. 
(a)

$$
\begin{aligned}
& \% \text { Fluorescence with } \\
& \text { continuous excitation }
\end{aligned}
$$

$(+/-$ S.E.M. , $n=31)$

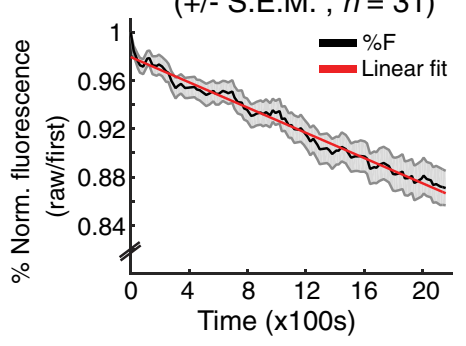

(d)

Single trial off ROI subtraction

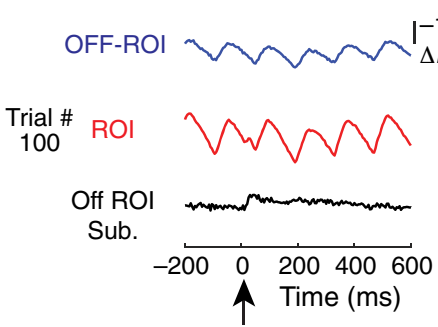

(b) Simultaneous imaging and blood flow recording $(n=1,1$ trial $)$

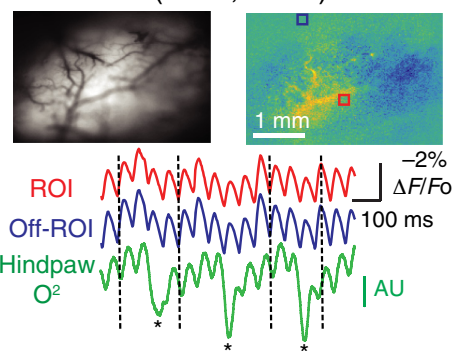

(e) Single trial spatial response trial \# 100

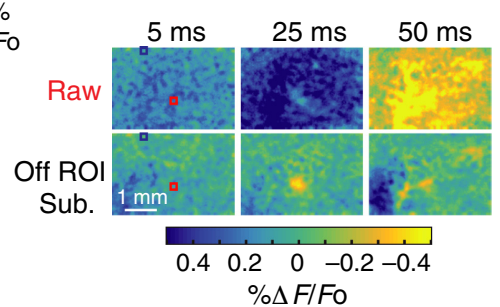

(c) ROI vs Off ROI signals $(n=1,102$ trials $)$

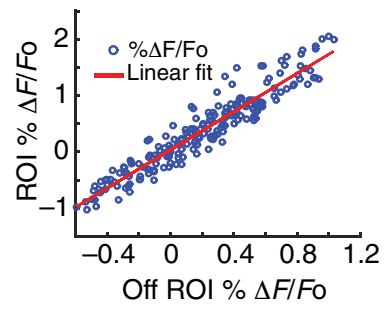

(f) Temporal mean response

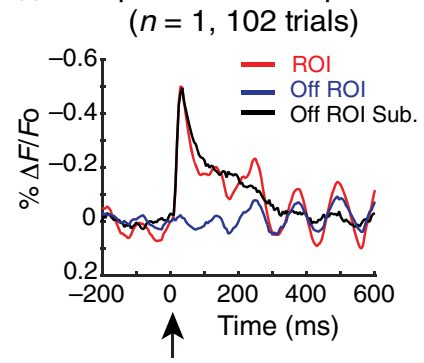

Fig. 3 Post hoc analytical methods for ArcLight widefield imaging. (a) Continuous 465-nm excitation of ArcLight causes slow decay of fluorescence over time. Each session was normalized to the first frame to compare responses across animals. Each imaging session was sampled every 6 to $8 \mathrm{~s}$ over $\sim 35 \mathrm{~min}$ and fit to a simple linear model to calculate the slope of decay (mean experiment $R^{2}=0.70, n=31$ ). (b) Normalized $\% \Delta F / F_{0}$ single frame and representative temporal traces show hemodynamic signal. Blood vessels are clearly seen outlined in yellow. Regions of interest (ROls) separated by $\sim 1 \mathrm{~mm}$ show highly correlated signal during nonstimulated trials. Simultaneously recorded blood oxygenation (green) taken from the hindpaw shows a similar phase shifted signal matching the ROI (red) and Off-ROI (blue) response. Asterisks $\left({ }^{*}\right)$ highlight times of large artifacts and potential respiration. Black guidelines help visually determine alignment of the signals. (c) Prestimulus $(200 \mathrm{~ms})$ normalized $\left(\% \Delta F / F_{0}\right)$ period shows highly correlated signals with a linear relationship $\left(R^{2}=0.89,1\right.$ trial). Prestimulus fitting was used to generate a model for ongoing activity for subtraction. (d) Example trials comparing raw Off-ROI \% $\Delta F / F_{0}$ (blue), $\mathrm{ROI} \% \Delta F / F_{0}$ (red), and the Off-ROI subtraction (black) method. Stimulus given at time $t=0$, black arrow. (e) Comparison of raw and Off-ROI subtracted single frames taken from trial 100 [(d) top]. Off-ROI subtraction shows clear reduction in the hemodynamic response. (f) Mean response between the raw ROI (red), raw Off-ROI (blue), and Off-ROI subtracted (black) method shows similar temporal averages ( $n=1,102$ trials). Clear oscillations are still prevalent in raw averaged condition; however, these oscillations are absent in the Off-ROI subtracted condition.

To characterize the temporal dynamics of the evoked signal, we reduced the spatial information down to a single ROI corresponding to a mouse cortical barrel. A single ROI $150 \times 150 \mu \mathrm{m}$ square was selected as the area of maximal response to the whisker deflection [see box outlined at $35 \mathrm{~ms}$, Fig. 2(a)]. The following analysis was conducted on the average response in each experiment (containing 50 to 100 trials). For every experiment, an Off-ROI was selected, scaled, and subtracted from the ROI to reduce ongoing hemodynamic noise (see Sec. 2.11 and Fig. 3 for more details of this procedure). A representative temporal response from the ROI during a single imaging experiment is shown in Fig. 2(b) [grand average Fig. 2(c)]. In order to provide the best estimate of the temporal parameters based on our sampling frequency $(200 \mathrm{~Hz})$, we approximated the measurements using linear interpolation and approximated the signal corresponding to the observed frame. The average signal onset, defined as the poststimulus time corresponding to the first frame reaching $10 \%$ of the maximal value, was determined to be $15 \pm 5 \mathrm{~ms}$ (SD). We calculated the average time from stimulus presentations to $50 \%$ and $90 \%$ of the peak response as $20 \pm 5 \mathrm{~ms}$ and $30 \pm 10 \mathrm{~ms}$ (SD), respectively (across experiments, $n=31,7$ animals). The mean ArcLight cortical response signal reached peak intensity at $35 \pm 15 \mathrm{~ms}(\mathrm{SD})$ poststimulus with a mean peak response of $-0.51 \pm 0.24$ (SD) $\% \Delta F / F_{0}$. Given the intrinsic ArcLight fluorophore reported rise-time, time between onset and peak, of 10 to $20 \mathrm{~ms},{ }^{1,2,18}$ our observations here correspond well with published in vivo anesthetized cortical extracellular single unit activity in layer 2/3 (Ref. 39) and simultaneously recorded LFP signals (see Fig. 4). Upon reaching peak, the signal decayed back to baseline over a highly variable range from 5 to $300 \mathrm{~ms}$. Across all animals [Fig. 2(c), $n=31$ experiments, 7 animals], the mean decay rate to $50 \%$ and $25 \%$ of maximal response was $95 \pm 105 \mathrm{~ms}$ and $245 \pm 200 \mathrm{~ms}$ (SD) [median: 50 and $155 \mathrm{~ms}$ ], respectively. A smaller secondary activation typically occurred $\sim 100$ to 200 ms poststimulus [Figs. 2(b) and 2(c)]. A large secondary onset was found only in $\sim 25 \%$ of experiments, and was defined as a period of rising activity for a duration of $\sim 100 \mathrm{~ms}$ poststimulus, and has been shown in widefield recording using VSDs. $^{40}$

The determined amplitude of the average evoked $\Delta F / F_{0}$ ArcLight response is similar to other reported voltage sensors' 
(a) Mean spatial responses ( $n=1,100$ trials)

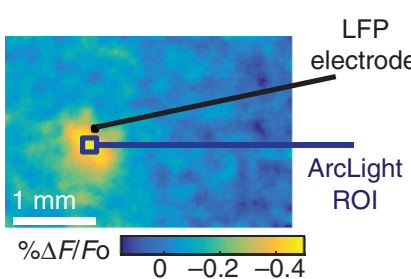

(d)
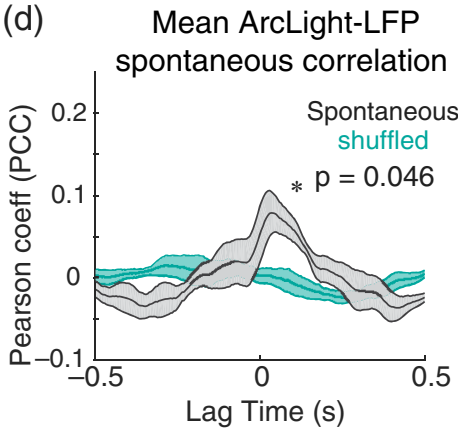

(b) Mean evoked response ( \pm SEM, $n=1,100$ trials) (c) Median temporal parameters $(n=5)$

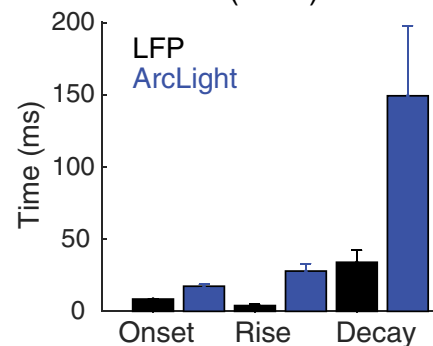

(f)

Single trial response

(e) Mean ArcLight-LFP

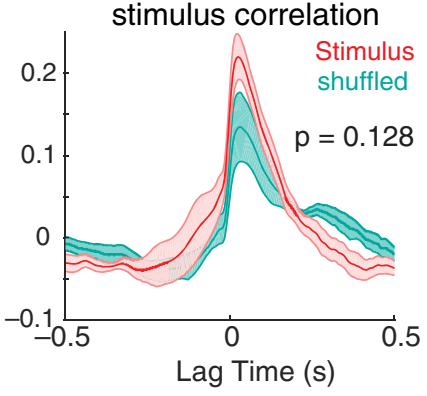

amplitude correlation

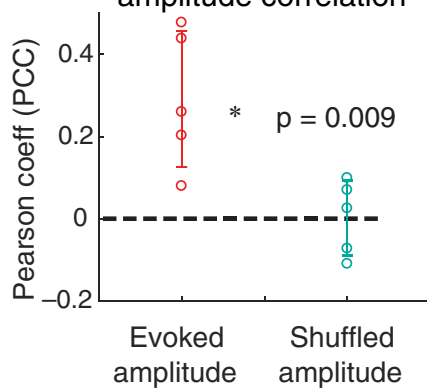

Fig. 4 Simultaneous paired extracellular LFP and ArcLight fluorescent recordings. (a) Spatial average fluorescent response (100 trials) to strong whisker deflection 25-ms poststimulus showing the ROI for peak ArcLight signal (captured at $200 \mathrm{~Hz}$ ) and the LFP location (captured at $2 \mathrm{kHz}$ ). All GEVI data shown in this figure have also been postprocessed using the Off-ROI subtraction method shown in Figs. 3 and Fig. 11 (see Sec. 2.11). (b) An example of simultaneously captured mean LFP (black) and ArcLight (blue) response $( \pm$ SEM) to single punctate whisker deflection ( $n=1,100$ trials). Note that LFP and ArcLight signals have been inverted. (c) Comparison between the temporal characteristics of the two signals. Note the similarly delayed $20-$ to $30-\mathrm{ms}$ onset and rise times compared to the LFP signal, and dramatically longer decay observed in ArcLight responses $(n=5, \pm$ SEM). (d) Mean spontaneous correlation between the simultaneously recorded LFP and the measured ArcLight response shows weak but significant correlation $(n=5,100$ trials each \pm SEM). $P$-value for the max correlation $(p=0.046$, at 35 -ms lag, unpaired $t$-test). (e) Mean correlation across single-trial responses between simultaneous LFP and ArcLight ( $n=5,100$ trials each). Stimulus (red) and trial shuffled (turquois) condition show similar correlation between signals suggesting this correlation is driven by common inputs. $P$-value for the max correlation ( $p=0.128$ at 35-ms lag, unpaired $t$-test). (f) Pearson correlation coefficient (PCC) between peak amplitudes (across a 20-ms window) of LFP and ArcLight response shows significant differences between the stimulus and trial shuffled condition ( $n=5$ experiments, $p=0.009$, unpaired $t$-test).

average peak responses in the $\mathrm{S} 1$ barrel cortex [mean evoked amplitudes, VSFP 2.3: $0.79 \% \pm 0.21 \% \Delta R / R,{ }^{23}$ RH1691: $0.70 \% \pm 0.4 \% \Delta F / F_{0},{ }^{23}$ and $\mathrm{RH} 1691: 0.26 \% \pm 0.11 \%$ $\Delta F / F_{0}{ }^{21}$ ] regardless of imaging setup, sampling rates, and anesthesia. Note even the same sensor (e.g., RH1691) ${ }^{20,21}$ has produced a wide range of reported amplitudes and varies heavily on experimental preparations. The average peak amplitude of the evoked response is similar, but reduced compared to in vivo ArcLight responses from the olfactory bulb (reported $1.2 \% \pm 0.05 \Delta F / F_{0},{ }^{18}$ sampled at $125 \mathrm{~Hz}$ ). The spatial and temporal results are also similar to published wide-field imaging responses using organic VSDs [RH 1691, ${ }^{20,21,32}$ and RH 795 (Ref. 40)] as well as other GEVIs [VSFP 2.3 (Ref. 23)]. Taken together, this evidence demonstrates that on average ArcLight is able to resolve sensory evoked cortical responses that are comparable to other voltage sensors.

One key benefit of wide-field imaging using voltage sensors is the ability to resolve functionally relevant cortical structures. As an initial validation, in one experiment, we compared the spatial component of the ArcLight activation to that obtained through conventional intrinsic imaging [see Sec. 2.11, Fig. 2(d), left panel ArcLight, right panel intrinsic imaging]. This resulted in good topographical correspondence between the two approaches. We further recorded spatial activity using ArcLight when stimulating multiple individual whiskers to generate an activity map of the barrel cortex. We stimulated surrounding whiskers one-by-one using the precise galvanometer while recording the evoked sensory response using ArcLight. We found that multiple whisker representations could be isolated across the barrel cortex [Fig. 2(e)]. When we superimposed these cortical activation regions, the resulting ArcLight responses correlated well with the stereotaxic alignment of a typical histological barrel map (data not shown).

\subsection{ArcLight Shows Slow Rate of Photobleaching In Vivo}

To achieve the fidelity of imaging presented in Fig. 2, we developed several analytical tools that are described in-depth here. ArcLight has been shown to be very photostable over long periods of excitation in vitro ${ }^{12,13,18}$ and therefore is ideal for long imaging experiments. We also found this to be the case here, where ArcLight showed only a small, slow linear decay of fluorescence over time. We determined this by continuously 
exposing the cortical surface to constant blue (465 nm) excitation during each imaging experiment ( $35 \mathrm{~min})$ and applied either a whisker stimulus or no stimulus. During the no-stimulus portion, we calculated the mean fluorescence in the selected ROI and fit a linear model to predict the decay over time. The average slope of the linear decay of the ArcLight was found to be a $0.3 \% \pm 0.24 \%$ standard error of the mean (SEM) change in fluorescence per minute $[n=31$ experiments across 7 mice; mean $R^{2}$ value $=0.69$, Fig. $\left.3(\mathrm{a})\right]$, with $400 \mathrm{~mW} / \mathrm{cm}^{2}$ LED system, Sec. 2.4]. Based on our optics, this LED excitation corresponds to an approximate $40 \mathrm{~mW} / \mathrm{cm}^{2}$ (or $0.4 \mathrm{~mW} / \mathrm{mm}^{2}$ ) overall intensity. The overall result of the slow rate of photobleaching appears to be consistent with in vitro findings. ${ }^{2}$ We subsequently accounted for the ArcLight photobleaching decay, as well as differing amounts of overall baseline fluorescence, by normalizing each frame as a percent over the baseline response $\left[\% \Delta F / F_{0}\right]$. This approach has been widely used in fluorescence imaging as a method to normalize and compare across animals. ${ }^{41}$ In this work, we define our baseline fluorescence $\left(F_{0}\right)$ as the single frame when the stimulus was delivered.

\subsection{Reducing Hemodynamic Signal with Post Hoc Off-ROI Subtraction Analysis}

In addition to the decay caused by the photobleaching, we observed large fluctuations in the fluorescence signal at frequencies consistent with hemodynamics [Fig. 3(b), 7 to $10 \mathrm{~Hz}$ ]. Hemodynamic signal is a common feature of blue-green light excitation fluorophores due to the overlapping absorption spectra of hemoglobin. ${ }^{19,42}$ We directly measured the hemodynamic signal in the fluorescence imaging using simultaneous recording of the blood oxygenation with a custom developed blood oxygenation sensor on the mouse's hindpaw (see Sec. 2.6). We determined that the hemodynamic signal was moderately correlated at fixed $30 \mathrm{~ms}$ delay (mean Pearson correlation: 0.54 $( \pm 0.16 \mathrm{SD})$, across 102 trials) with changes in blood flow and oxygenation in the hind-paw. The general single-trial pattern of activity between the two signals showed good correspondence [Fig. 3(b)], suggesting that the observed oscillatory signal was likely due to the overlapped excitation frequency $(465 \mathrm{~nm})$ between the ArcLight fluorophore and hemoglobin (see Appendix A3). Although the blood oxygenation signal is similar to the hemodynamic signal in the voltage fluorescence imaging, we observed differences in the two signals, specifically in the introduction of larger artifacts likely due to respiration [Fig. 3(b), black asterisks $(*)$ ] that were absent in the fluorescence response. Therefore, we sought additional methods as a model to remove the ongoing hemodynamic response in the fluorescent signal. A simple notch filtering at the heartbeat frequency $(7$ to $10 \mathrm{~Hz})$ dramatically distorted and reduced the evoked ArcLight response and was thus not a viable approach (see Appendix A1).

To counter the hemodynamic interference, we instead developed a simple post hoc linear model to take advantage of the highly correlated nature of the hemodynamic signal in the fluorescence signal across pixels. Using this method, we extracted single-trial information by subtracting a linear projection of an Off-ROI signal from the ROI time series. The Off-ROI was selected as a highly correlated region at least $1 \mathrm{~mm}$ away from the ROI, as defined from the evoked response [Figs. 3(b) and 3(c), Sec. 2.11, mean distance between ROI and Off-ROI: $1.35 \mathrm{~mm}$, range of distance: 1.15 to $1.85 \mathrm{~mm}$ ]. The Off-ROI region was always placed further than the $50 \%$ contour of the maximal sensory evoked response (mean max evoked radius: $0.60 \pm 0.261 \mathrm{~mm}$ ). To avoid subtracting stimulus information, we used only $200 \mathrm{~ms}$ of prestimulus activity to determine the corresponding coefficients of the projection. Similar to other widely used subtraction methods, ${ }^{43}$ there is a potential of the introduction of neural responses and the negation of common brain states. Despite these limitations, we found this Off-ROI subtraction technique was suitable for our purposes of measuring the relative evoked activity caused by the sensory stimulus.

Using both fluorescence normalization and Off-ROI subtraction, we dramatically reduced the ongoing noise and improved the single-trial signal-to-noise ratios (SNRs) [Fig. 3(d), singletrial example; Fig. 3(f), trial averaged]. Qualitatively, we observed that the Off-ROI subtraction significantly reduced the hemodynamic component of the ArcLight signal, without compromising the evoked response, seen in both time series and spatial representations [Figs. 3(d), 3(e), for details see Appendix A2, Figs. 9 and 10]. To better quantify the reduction in noise, we assessed the ability to detect evoked responses from the ArcLight signal with and without Off-ROI subtraction. Here, we measured single-trial SNR by comparing the mean evoked response between 25 and $30 \mathrm{~ms}$ poststimulus to the variability of ongoing noise, across all sessions $(n=31$ experiments, 3008 single trials). The noise $(\mathrm{N})$ was defined as the mean standard deviation of the ArcLight signal over the 200-ms window before stimulus onset. We found that with trial averaging the ArcLight response could be detected [mean response SNR: $11.63( \pm 9.5$ $\mathrm{SD}$ ); however, single trials were too embedded in the noise to be clearly separated [single-trial SNR: 0.99 ( \pm 1.64 SD)]. In contrast, the Off-ROI subtraction method dramatically increased both mean $[30.0( \pm 27.37 \mathrm{SD})]$ and single trial [4.02 ( \pm 1.93 SD)] SNRs [Figs. 3(d), 3(f)]. The ArcLight SNR of the average response (with and without Off-ROI subtraction) is comparable to other voltage indicators, (VSFP Butterfly 1.2). ${ }^{19}$ However, without Off-ROI subtraction, the raw singletrial SNR is noticeably worse than the reported single-trial SNR values in other GEVIs [VSFP Butterfly 1.2, ${ }^{19}$ and VSFP 2.3 (Ref. 3)]. However, it is important to note that these fluorescence resonance energy transfer (FRET)-based GEVIs utilize post hoc ratiometric subtraction methods to improve SNR. By gaining access to single-trial information with Off-ROI subtraction, we dramatically increase the usability for wide-field imaging of ArcLight in behaviorally relevant contexts.

\subsection{Comparison of ArcLight Response to Simultaneously Recorded Local Field Potential}

In order to validate ArcLight as a correlate of neural activity, we simultaneously measured the LFP while imaging the cortical response to punctate whisker deflections (Fig. 4). Based on the histological analysis and the limitations of blue light excitation, ${ }^{44}$ we expect that the ArcLight response is predominantly from layer 2/3 neurons (Fig. 1). Thus, we inserted a low impedance electrode $\sim 250$ to $300 \mu \mathrm{m}$ below the cortical surface near the centroid of the evoked response and simultaneously recorded the corresponding LFP during an anesthetized imaging experiment (see Sec. 2.7). The following data represent comparisons between the simultaneously recorded ArcLight and LFP responses (for details see Sec. 2.7). The LFP signal has been notched filtered at $60 \mathrm{~Hz}$.

We compared the resulting stimulus evoked responses in the LFP and the evoked fluorescence and found similar characteristics between the two signals, shown in Fig. 4(b). Specifically, 
we found that the average LFP and ArcLight responses during whisker stimulation were correlated [mean peak Pearson correlation: 0.65 ( $\pm 0.118 \mathrm{SD})$ ] ( $n=5$ experiments, across 2 mice). Furthermore, we determined the peak correlation between the LFP, and the ArcLight response was $35 \mathrm{~ms}( \pm 20 \mathrm{~ms}$ SD, median: $25 \mathrm{~ms}$ ), delayed relative to the LFP signal [Fig. 4(b)]. Note that the determined ArcLight peak response was highly variable (range 25 to $100 \mathrm{~ms}$ ). During the simultaneous ArcLight-LFP experiments, the average peak response was $70 \pm 20$ (SD) milliseconds poststimulus (median: $60 \mathrm{~ms}, n=5$ paired recordings, across 2 mice).

We directly compared the difference in temporal dynamics between the evoked LFP and the cortical ArcLight responses [Fig. 4(c)] by measuring the onset, $10 \%$ to $90 \%$ rise time, and the $50 \%$ decay time for the simultaneously collected LFP and ArcLight signals. Note that the relationship between the LFP and the membrane potential is quite complex (for review see Ref. 45), where the exact coupling between the LFP and the membrane potential is still being discovered. ${ }^{46}$ Other work has suggested that the LFP is an approximation of the temporal derivative of the membrane potential. ${ }^{47}$ However, more recent works have noted the potential influence of filtering properties on relating the LFP to the membrane potential, and thus caution needs to be used in interpreting this relationship. ${ }^{48}$ Our comparison between the temporal dynamics of the LFP and the ArcLight response may not account for this complex relationship between the LFP and the membrane potential.

We found that in general, the response onset [mean onset ( \pm SD) LFP: $8.6(0.75) \mathrm{ms}$, ArcLight: $20(5) \mathrm{ms}$ ] and rise time [mean rise time ( \pm SD) LFP: 4.7 (1.7) ms, ArcLight: 30 (15) ms] of the evoked cortical ArcLight signal were 10 to $25 \mathrm{~ms}$ later than the LFP response [Fig. 4(c)]. However, the mean ArcLight signal decay time was prolonged relative to the LFP decay time [mean decay $( \pm \mathrm{SD})$ LFP: 35.5 (18.5) ms, ArcLight: 170 (108) ms]. This long excitatory tail has been a characteristic of other voltage sensitive imaging techniques, including VSDs, ${ }^{40}$ which have substantially faster temporal dynamics, as well as other GEVIs including VSFP 2.3. ${ }^{23}$ Therefore, this slow decay from the initial response may not entirely represent limitations of the molecule, but potentially additional physiologically relevant information. An alternative possibility is that the long tail is a hemodynamic artifact that has not been removed with the Off-ROI subtraction technique or is part of an intrinsic hemodynamic response. However, due to the prevalence of this long tail in other published sensors, ${ }^{20,23}$ it is likely that the prolonged fluorescence response represents prolonged excitation caused by a strong sensory stimulus. These data suggest that at the least the average ArcLight signal represents the average fast transients present in the LFP.

In addition to comparing the mean LFP and ArcLight response, we determined how well the ArcLight signal captured the single-trial LFP response [Figs. 4(d)-4(e), $n=5$ whiskers, across 2 mice, 100 trials each]. In a single trial, we measured the correlation between the ongoing LFP signal and the resulting ArcLight fluorescent response. We found that the spontaneous LFP and ArcLight responses were only weakly correlated periods of quiescence, Fig. 4(d), [maximum average Pearson coefficient spontaneous: $0.076(0.067 \pm \mathrm{SD})$ at $35 \mathrm{~ms}$ poststimulus], that was significantly different compared to a trial shuffled case [mean Pearson correlation shuffled spontaneous, 0.002 ( \pm 0.02 SD), $p=0.046$, unpaired Student- $t$ test]. Although the single-trial correlation between LFP and ArcLight is low, these results are consistent with the weakly correlated singletrial LFP and similar wide-field voltage sensitive imaging techniques. ${ }^{33}$ During stimulation periods, Fig. 4(e), we also found that the LFP and the ArcLight were slightly more correlated [mean Pearson correlation stimulus: $0.22( \pm 0.063 \mathrm{SD})$, temporal lag of $35 \mathrm{~ms}$ ], shown in Fig. 4(e) (red trace). However, when we shuffled the trials to determine the correlative effects from the input, the correlation between shuffled and unshuffled signals was very similar [mean Pearson correlation shuffled stimulus: 0.13 ( $\pm 0.10 \mathrm{SD}), p=0.128$, unpaired $t$-test], suggesting that the correlation observed during stimulation was predominantly associated with the strong evoked response.

Instead of simply correlating the entire signal, we focused our analysis on determining if the LFP signal and the ArcLight evoked stimulus response amplitudes covaried. Here, we define the single-trial response amplitude as the difference in activity between the signal preceding the stimulus and maximum response within a 20 -ms window during each imaging session's peak response [Fig. 4(f)]. By limiting the analysis to the evoked peaks, we determined that the evoked response amplitudes between the two signals were correlated [mean Pearson correlation amplitude: $0.29( \pm 0.17 \mathrm{SD})]$. Moreover, when we shuffled the trials, this correlation between the response amplitudes disappeared [mean Pearson correlation shuffled amplitude: $-0.0018( \pm 0.09 \mathrm{SD}), p=0.009$, unpaired $t$-test]. These data suggest that the ArcLight and LFP amplitudes weakly covary in response to a sensory input. Taken together, these results suggest that ArcLight has the capacity to capture evoked features similar to evoked LFP and potentially provides additional information on ongoing cortical processes.

\subsection{ArcLight Cortical Response to Complex Stimuli}

Given the relatively long decay of the signal as measured by ArcLight in response to a single punctate sensory stimulus, this naturally begs the question as to the nature of the response to more complex inputs. To determine the temporal capabilities of ArcLight to represent complex stimuli, we presented a range of inputs to the whisker and recorded the downstream evoked cortical fluorescent response. We selected complex inputs that have been commonly used in the rodent vibrissa system, ${ }^{39,49-52}$ and therefore, these stimuli represent an additional comparison to published traditional electrophysiological recordings. Again, mice were anesthetized under low isoflurane (Sec. 2) and stimulated using a high-fidelity galvanometer device on a single whisker $10 \mathrm{~mm}$ from the face. We presented a range of sensory inputs from a simple ramp-and-hold ${ }^{53,54}$ to pulsatile frequency deflections $(2.5$ to $40 \mathrm{~Hz})$ and recorded the evoked ArcLight response [Fig. 5(a), $n=3$ whiskers, across 2 animals]. Each trace represents the average response (100 trials per animal, 3 animals) within a single ROI taken as the maximal response $25 \mathrm{~ms}$ after stimulus presentation. In response to the ramp-and-hold stimulus, the cortical ArcLight response shows two clear peaks of activity corresponding to the rising $(\mathrm{ON})$ and falling (OFF) stimulus events [Fig. 5(a), panel 1]. The two ON and OFF sensory peaks are representative of a well-documented velocity sensitivity of the rodent whisker somatosensory pathway. ${ }^{30,39,53}$ Additionally, we found that ArcLight S1 cortical responses clearly resolved repetitive frequency-dependent inputs between the ranges of 2.5 to $20 \mathrm{~Hz}$ [Fig. 5(a), panels 2 to 5], with evoked peaks of activity corresponding to the presented sensory stimulus. However, 


\section{(a)}

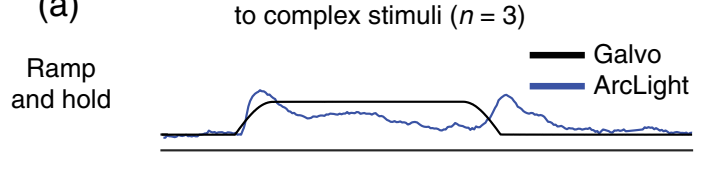

$2.5 \mathrm{~Hz}$

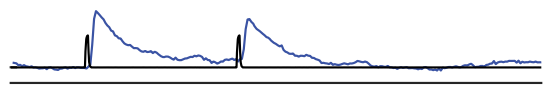

$5 \mathrm{~Hz}$

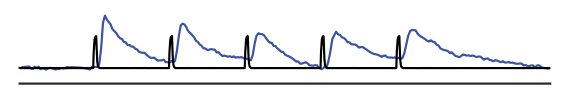

$10 \mathrm{~Hz}$

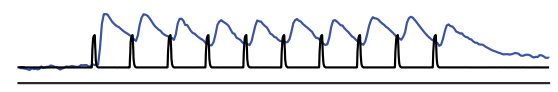

$20 \mathrm{~Hz}$

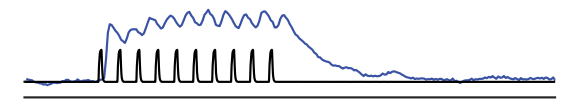

$40 \mathrm{~Hz}$

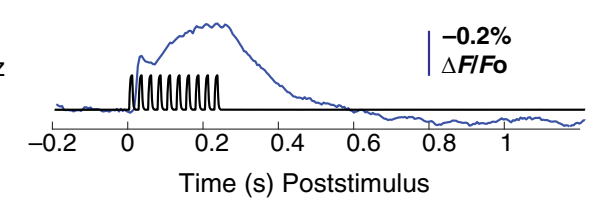

(b)

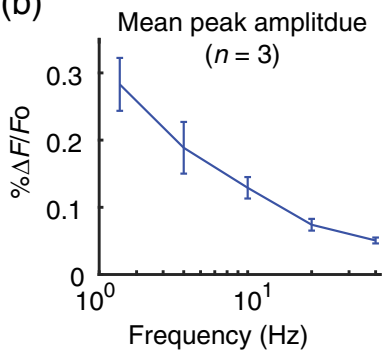

Fig. 5 ArcLight response to complex stimuli. (a) All GEVI data shown in this figure have been postprocessed using the Off-ROI subtraction method shown in Figs. 3 and 11 (see Sec. 2.11). Each panel shows the temporal response taken from a single ROI during specific complex whisker stimulation $(n=3)$. Each panel shows the corresponding galvanometer input (black trace), and the corresponding ArcLight (blue trace) output response. ArcLight shows clear response from 2.5 to $20 \mathrm{~Hz}$; however, ArcLight is unable to clearly resolve whisker inputs of $40 \mathrm{~Hz}$. (b) Mean peak amplitude for each stimulus taken as the difference between the evoked peak fluorescence and the fluorescence preceding each pulse.

high-frequency [Fig. 5(a), panel 6, $40 \mathrm{~Hz}$ ] deflections produced an overall increase in fluorescence that failed to clearly follow the sensory input. These results are summarized in Fig. 5(b), showing the amplitude of the peak ArcLight response as a function of stimulus frequency. The high-frequency $40-\mathrm{Hz}$ stimulation is at the upper limit of the innate capabilities of ArcLight based on in vitro studies. ${ }^{2}$ These in vitro ArcLight experiments demonstrate fast temporal dynamics with a 10 - to 20 -ms rise time, ${ }^{12,18}$ and $\sim 20 \mathrm{~ms}$ decay, which limits the fluorophore's ability to represent high-frequency information greater than $40 \mathrm{~Hz}$. Note that few studies have specifically examined the frequency encoding in mice under isoflurane anesthesia, so the limitations from a coding perspective are presently unclear. Taken together, these data demonstrate the use of ArcLight as a measurement of complex stimuli and frequency content in the S1 barrel cortex and highlight the potential limitations to resolve high-frequency information.

\subsection{Stability and Variability of ArcLight as a Measure of Cortical Response}

One critical component of any imaging fluorophore is the stability of the observed response over time. Our goal was to determine if the ArcLight cortical response dramatically changed during a long imaging experiment with continuous excitation. In a single trial, we would expect differences in activation of the barrel cortex due to various sources of neural variability, ${ }^{55,56}$ however, the average response over many trials will ideally remain relatively consistent during each experiment.
Here, we analyzed the single-trial evoked sensory response during an imaging session after removing the shared hemodynamic signal using the post hoc Off-ROI subtraction method. Figure 6(a) shows the peak response frame averaged across trials for a single whisker deflection. For this analysis, we limited our investigation of stability to the temporal component of the main ROI of a single mouse barrel [Fig. 6(a), red square, $150 \times 150 \mu \mathrm{m}]$. For the outlined ROI, Fig. 6(b) shows the time series of fluorescence on a trial-by-trial basis over 102 trials. Notice, on single trial there is trial-to-trial variability [Fig. 6(b): 102 trials, Fig. 6(c), top: 25 sequential trials]. However, when we average over blocks of 25 trials, the evoked signals appear to be quite similar, shown in Fig. 6(c), bottom.

We evaluated the stability within an imaging experiment by measuring the resulting distribution of responses within blocks of 25 sequential trials $(\sim 600 \mathrm{~s})$. In this analysis, we included only one (the first) imaging experiment from each animal $(n=7)$ to avoid skewing the results with data from a single mouse. Each experiment was normalized to the mean peak response for comparisons across animals. Specifically, we measured the peak response amplitude within a 20-ms window [Fig. 6(d), left]. We compared the resulting distributions of single-trial response amplitudes between the first trial block ( $\sim 525 \mathrm{~s})$, and last trial block ( 2150 s) of the experiment across each animal [Fig. 6(d), right]. We found that within all experiments $(7$ mice) the difference between single-trial response amplitudes between the first and last 25 trial blocks were statistically insignificant [Fig. 6(d), right, $p>0.05$, paired 
(a) Single experiment response

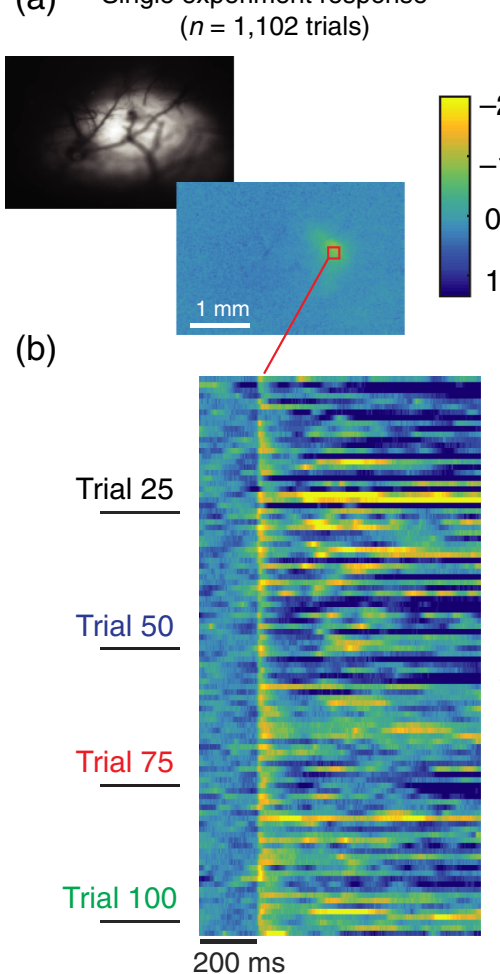

(c)

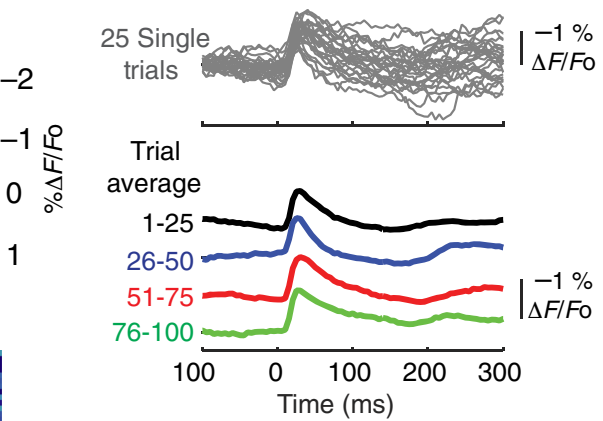

(d)

Mean peak amplitude within moving average (+/- S.E.M , 25 trial blocks)

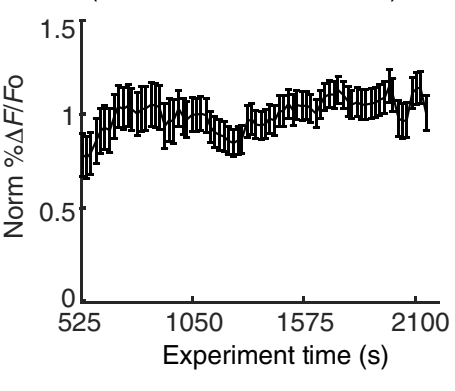

Mean peak amplitude across mice $(n=7)$

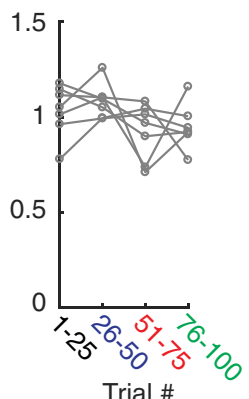

Fig. 6 Within experiment variability of ArcLight responses to single whisker deflection. Note that all GEVI data shown in this figure have been postprocessed using the Off-ROI subtraction method shown in Figs. 3 and 11 (see Sec. 2.11). (a, left) Raw fluorescence image of the ArcLight expression. (a, right) example of the average cortical response (102 trials) of a $1200 \mathrm{deg} / \mathrm{s}$ single whisker deflection. The spatial activity is reduced to a single response of a $9 \times 9$ pixel $(\sim 150 \times 150 \mu \mathrm{m})$ area for subsequent analyses. (b) Single-trial variability of the ArcLight response. Stimulus onset at $200 \mathrm{~ms}$. Each row represents a single trial in an entire session, where each column represents the $\mathrm{ROI}$ at a single frame (200-Hz frame rate). (c, top) Single-trial time series of the first 25 sequential trials. On a single trial, the evoked response is quite variable. (c, bottom) The average response is quite stable over time as determined by the similarity of the 25 -trial averages. (d, left) Mean peak amplitude $( \pm$ SEM) of the response in a 25-trial moving average over an entire imaging session. (d, right) Mean responses across all mice $(n=7)$ in 25 trial blocks during imaging session. Across all animals the first and last trial blocks were not significantly different $(n=7, p>0.05$ paired $t$-test).

$t$-test]. These results suggest that across the entire imaging experiment the peak response amplitude remained highly consistent.

In addition to the stability of the ArcLight response, we also determined the within experiment variability of the evoked response, regardless of any temporal drift. Similar to published VSD recordings, ${ }^{57}$ ArcLight exhibited high variability in fluorescent responses to a simple stimulus, shown as a 25 -trial example in Fig. 6(c) (102 trials, 1 animal). We used the methods described above and averaged the fluorescent response across a moving 25-trial window to track parameters over time. We found that the within-experiment ArcLight response amplitude varied by $12.2 \%$ [ $\pm 4.6 \mathrm{SD}$ across 7 mice], measured as the standard deviation across an entire imaging experiment. These data suggest that the ArcLight cortical response is relatively stable across a long imaging experiment and exhibit variability that is consistent with other measurement modalities.

\subsection{Repeatability of ArcLight Recording over Multiple Days}

One of the great benefits of genetically expressed voltage probes is the ability to record from an animal over many days, weeks, and months. Repeatedly imaging over many days dramatically increases the data gained from a single animal, which may be of particular importance for behavioral experiments where mice need to be trained over weeks or months. To test the repeatability of the ArcLight response, we recorded the evoked fluorescence to the deflection of the same single whisker over a series of days $(1,3,5,7,14,21,28)$. Each imaging experiment lasted $\sim 1$ to $2 \mathrm{~h}$. We controlled isoflurane levels through constant measurement of physiological parameters (mainly heart rate, see Sec. 2.8) to minimize effects of different depths of anesthesia across days. Furthermore, we always attempted to stimulate the same whisker across imaging experiments. During one imaging experiment (mouse 2, day 14), the animal's target whisker was not present, and therefore, we imaged the response to a different whisker in this isolated case.

We found that we were able to record the evoked responses over the course of 28 days [Fig. 7(a), $n=3$ mice]. After 28 days, the fluorescence response was still clearly visible, suggesting additional time-points could continue. Over the course of a month, the resulting spatial [Figs. 7(a) and 7(d)] and temporal dynamics [Fig. 7(b)] were consistent within an animal across days to weeks. Across all experiments, we found that the peak response amplitude (measured as normalized to day 
(a) ArcLight cortical response across repeated imaging sessions

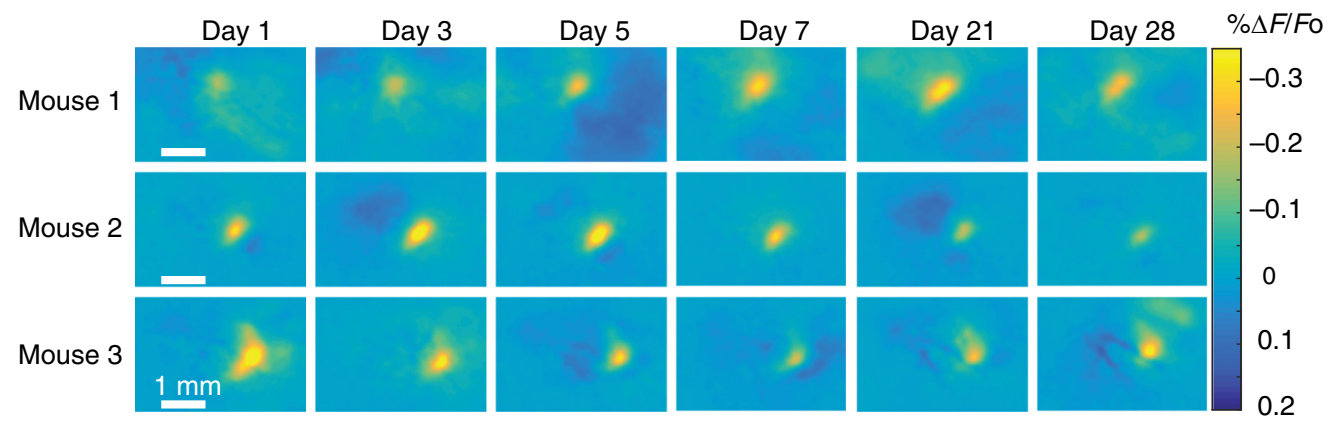

(b)

Multiple day mean ROI timeseries (102 trials)

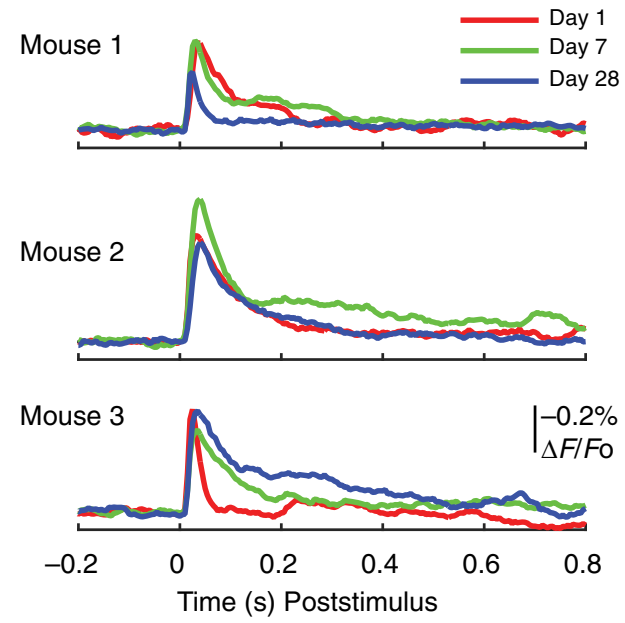

(c) -0.8 Mean peak response amplitude

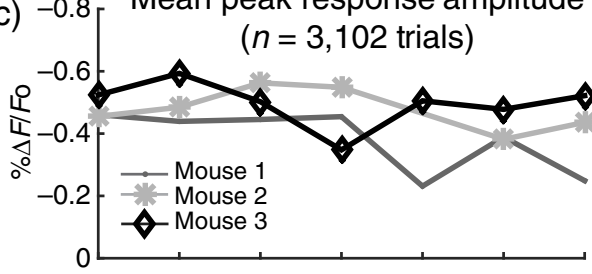

Peak response $\sqrt{\text { area }}$

(d) 600 (50\% contour, $n=3,102$ trials)

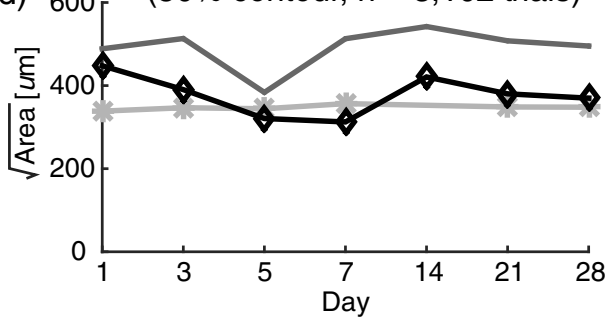

Fig. 7 Multiday imaging of ArcLight cortical response. Note that all GEVI data shown in this figure have been postprocessed using the Off-ROI subtraction method shown in Figs. 3 and 11 (see Sec. 2.11). (a) Each image represents the averaged Off-ROI subtracted $\% \Delta F / F_{0}$ cortical response between 25 and $35 \mathrm{~ms}$ postwhisker deflection over the course of 28 days (each day 102 trials). (b) Time series of the ROI on selected days ( 1 [red], 7 [green], 28 [blue]). The time series shows consistent waveforms across imaging sessions. (c) Evoked peak response amplitude across repeated days. Across all mice there is no apparent trend during the 28-day period. (d) The $50 \%$ contour of the evoked response seen in (a). In this panel, the square root of the area is displayed to show an intuitive measurement of overall size of the evoked response (assuming a $n \times n$ square). Again, there is no clear trend across the three mice during the 28-day period.

$\left.1 \Delta F / F_{0}\right)$ was relatively consistent during repeated imaging sessions [mean response amplitude: $96 \pm 18.3$ SD\% Norm $\Delta F / F_{0}, n=3$ mice over 7 imaging sessions]. Furthermore, we found that the response amplitude variability across sessions in the same mouse was slightly less than the observed variability across different mice, but greater than the variability within an imaging session (amplitude SD same mouse: 18.3\%, $n=3$, amplitude SD across mice: $23 \%, n=7$, amplitude SD within session, $12.2 \%, n=7$ ). Although we attempted to image under the same experimental conditions, the variability across days is expected to be higher than the within session variability due to slight changes in window quality, anesthesia level, and camera alignment. In order to assess the consistency of the spatial information, we compared the area of the evoked response across each day [Fig. 7(d)]. We measured the area of activation as the $50 \%$ contour of the peak response of the mean 25 to $35 \mathrm{~ms}$ poststimulus frames (Sec. 2). We found that the evoked cortical area to be relatively consistent across all repeated imaging experiment on the same whisker [mean area: 1.66 $\left( \pm 0.348\right.$ SD) e $5 \mu \mathrm{m}^{2}$, Fig. $\left.7(\mathrm{~d})\right]$. The evoked area variability
(SD) observed across repeated imaging experiments in the same mouse was less than the variability across mice and different whiskers (area SD across experiments same mouse: $21.0 \%$, $n=3$, area SD across mice, $41.4 \% n=7$ ). These results suggest that repeatable imaging of ArcLight is consistent over the many weeks and months.

Above, we considered variability independent of possible trends across days; however, in order to determine potential drift, we compared the resulting distribution of single-trial responses between the first day, and final day of imaging [mean shown in Fig. 7(b) and 7(c)]. We determined that a majority of the animals $(2 / 3)$ experienced insignificant differences between the 1 st day and 28 th day $(p<0.05$, paired Wilcoxon signed-rank test) of imaging. Additionally, we found no clear trend in the evoked area of the evoked response [Fig. 7(d), shown as the square root of the area]. Here, the evoked area 25 to $35 \mathrm{~ms}$ poststimulus in mouse 2 slightly increased, in mouse 3 slightly decreased, and in mouse 1 remained constant. Even under extreme care, it is difficult to definitively determine the origin of the change in evoked fluorescence considering the 
many different parameters including window quality, experiment prep, and anesthesia level across imaging experiments and across mice. Therefore, it is unclear whether this change in evoked response is due to changes of the fluorophore (and expression) over time. Taken together, the above results demonstrate the capabilities of ArcLight to capture spatial and temporal information over many weeks and months. However, based on the day-to-day variability, careful analysis must be conducted when comparing and combining responses across days.

In addition to the evoked response amplitude, we determined if temporal dynamics remained consistent across many days of imaging. As shown in a subset of days [Fig. 7(b)], the temporal dynamics appear to be highly consistent across imaging experiments. We measured the Pearson correlation coefficient (PCC) across each imaging experiment to determine the changes in temporal waveform of activation. Here, we found the PCC to be highly correlated across imaging days mean $0.753 \pm$ 0.128 . In some imaging experiments, slight changes in evoked response did occur, particularly $\sim 150-\mathrm{ms}$ poststimulus in the presence or absence of the secondary activation. However, the overall high correlation between evoked waveforms across weeks of imaging suggests that under highly controlled conditions, the same whisker stimulation produces very similar temporal activation.

\subsection{Awake Recordings of Evoked ArcLight Mean and Single-Trial Responses}

Finally, our goal was to determine if ArcLight had the capacity to represent cortical sensory responses in the awake rodent.
Although previous studies have shown awake response of ArcLight, these examples were in either different species (Drosophila) or systems (mouse olfactory bulb), which would not guarantee clear fluorescent responses in cortical structures. Again, mice were left to express for four weeks before imaging (see Sec. 2.9). Mice were habituated over a period of three days to withstand long sessions of headfixation (Sec. 2) but were not trained on any task. In order to prevent whiskers from slipping out of the whisker stimulator galvanometer, the device was placed $5 \mathrm{~mm}$ from the face. We applied a similar stimulus as shown in Fig. 2; however, the stimulus was adjusted for the adjusted distance to the face. We report that ArcLight reveals a robust sensory evoked response even under awake conditions in the S1 barrel cortex (Fig. 8). In Fig. 8, we have shown a representative sensory evoked S1 cortical response; however, we observed similar responses across imaging experiments (5 whiskers across 3 mice). In the awake animal, we observed a decrease in the evoked $\Delta F / F_{0}$ response with a corresponding decrease in the SNR $(2.43 \pm 0.92 \mathrm{SD}, n=5)$. During periods of no stimulus presented to the whisker, the average spatial and temporal responses were negligible, as expected [Fig. 8(a), top, Fig. 8(b)]. When the whisker was deflected with a $1200 \mathrm{deg} / \mathrm{s}$ pulse, the evoked response showed clear spatial and temporal activity similar to the anesthetized case [Fig. 2, as compared Figs. 8(a) and 8(b), single trials shown in Fig. 8(c)]. Due to the high variability of the ArcLight response, we would need substantially more data to make additional comparisons to the anesthetized case, and goes beyond the scope of this work. Taken together, this work highlights the ability of ArcLight as a robust spatial and temporal measurement tool of ongoing neural activity.

(a)

Awake mouse cortical ArcLight response

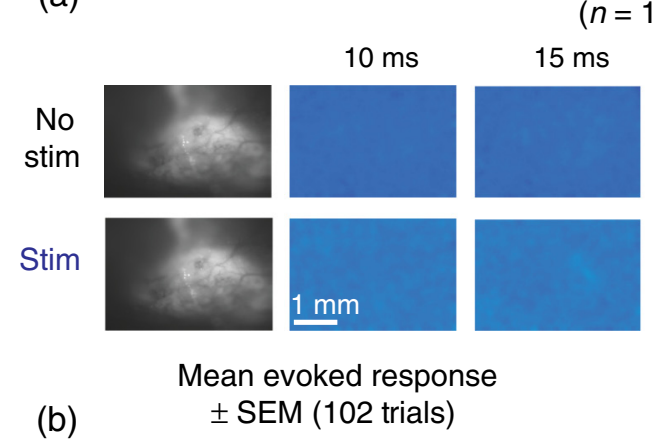
$(n=1,102$ trials)

(b)

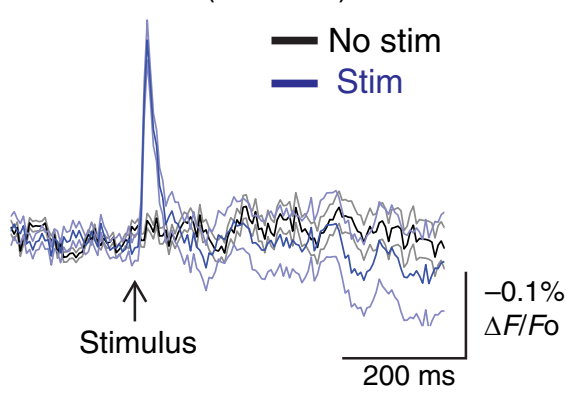

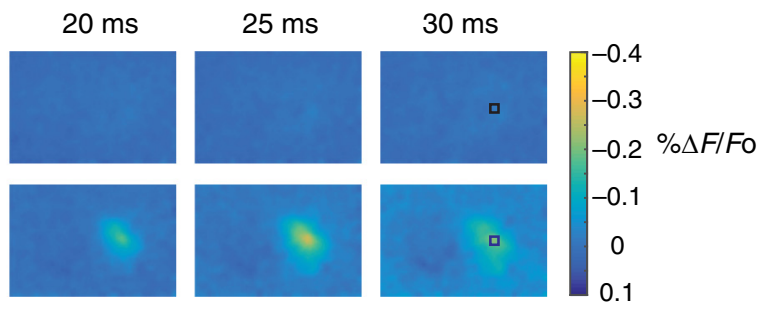

(c)

Sequential single trials $(n=1,25$ trials)

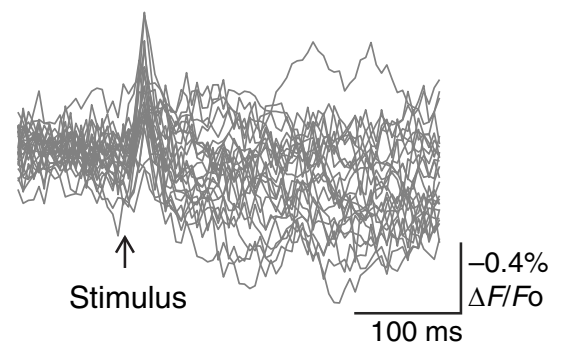

Fig. 8 Cortical ArcLight fluorescent responses in the awake mouse. Note that all GEVI data shown in this figure have been postprocessed using the Off-ROI subtraction method shown in Figs. 3 and 11 (see Sec. 2.11). (a) Mean fluorescent responses taken from the awake mouse (102 trials). (a, top) Average spatial response during no stimulus presentation. (a, bottom) Average spatial response during stimulus presentation (1200 deg/s punctate deflection). (b) Mean temporal fluorescent responses from the ROI [black square, $30 \mathrm{~ms}$ in (a)] with \pm SEM. (c) Example of 25 sequential single-trial responses taken from the same data set in the awake mouse. Clear stimulus evoked activity $\sim 20$ ms poststimulus. Stimulus presentation represented by black arrow. 


\section{Discussion}

In this work, we examined the functional characteristics of the genetically expressed voltage indicator (GEVI) ArcLight through testing in the widely used rodent sensory whisker pathway. With recent advances in GEVIs, there is a large demand to determine the in vivo functional limitations and capabilities for each new voltage probe. Since the initial discovery and publication; ${ }^{1}$ to our knowledge, ArcLight has been predominantly adopted as a tool for neuroscience research in Drosophila ${ }^{14,15,16,58,59}$ and has not been widely utilized in mammals. Other FRET-based GEVIs such as Butterfly 1.2, and VSFP 2.3 have been shown to measure widefield cortical responses in vivo; however, the monochromatic fluorophore ArcLight has yet been tested in in vivo cortical systems. We found that ArcLight produced a robust fluorescent response in the $\mathrm{S} 1$ barrel cortex in the anesthetized and awake mouse at high temporal and spatial resolution. We intend for this work not only to exclusively highlight the promise of ArcLight as a technique but also to provide a roadmap and a set of criteria for future GEVIs to be tested before widespread use.

\subsection{ArcLight Imaging as a Method for Measuring Cortical Activation on a Mesoscopic Scale}

Here, we used wide-field imaging to capture changes in population dynamics across the S1 barrel cortex on a mesoscopic scale (tens of $\mu \mathrm{m}$ ). Wide-field imaging is an alternative imaging modality that enables high temporal resolution recording across large structures on the order of cortical columns. ${ }^{60,61} \mathrm{We}$ found that ArcLight was able to provide clear and repeatable fluorescence responses spatially resolved at the level of a single cortical barrel on fast timescales (tens of ms, Figs. 2, 4, 6), which makes it well suited for investigations into sensory precepts.

One of the biggest concerns with wide-field imaging is the uncertainty of the origin of the neurological signal responsible for the recorded evoked fluorescence. Using GEVIs, such as ArcLight, we gain additional selectivity over traditional VSDs (e.g., RH1691) by specifically targeting only neuron membranes and avoiding the pharmacological effects of staining with dyes. ${ }^{62}$ In this work, we utilized the human synapsin 1 (hsyn1) promoter to express ArcLight in predominately layer $2 / 3$ and layer 5 neurons (Fig. 1). Additional genetic lines could reduce the uncertainty of expression to a single layer, or neuronal subtype, thereby increasing the utilization of GEVIs to measure specific in vivo circuit dynamics.

Similar to LFP, ECoG, and BOLD fMRI, the underlying neural correlate of the wide-field GEVI response represents a combination of electrophysiological sources. We directly compared simultaneous recordings of LFP and ArcLight fluorescence during sensory stimulation (Fig. 4) to determine the relationship between these two modalities. On average, the stimulus-evoked LFP and fluorescence were correlated with some differences in temporal dynamics. However, in a single trial, the ArcLight and LFP signals were weakly correlated even during large evoked sensory features. Although the single-trial relationships between these signals are weak, the results are similar to weak correlations found between LFP and VSD imaging. ${ }^{33}$ Furthermore, propagating waves of cortical activity traverse the cortical layers in complex patterns ${ }^{63}$ and may compound as dynamic signals in the in vivo fluorescent response that is not represented in the evoked LFP. Additionally, wide-field recorded ArcLight fluorescence is believed to represent a spatial measurement of neural membrane potential, ${ }^{1,18}$ which is fundamentally different from extracellularly recorded LFP. Furthermore, the relationship between the LFP and the membrane potential is quite complex (for review Ref. 45), with some work proposing that the LFP represents the first derivative of the ongoing membrane potential. ${ }^{47}$ However, this finding should be regarded with caution as the filtering properties have been shown to strongly influence the nature of the LFP and membrane potential relationship. ${ }^{48}$ In paired intracellular and LFP recording experiments, LFP explains only a limited amount of the signal variance in the membrane potential. ${ }^{46}$ Finally, due to the large hemodynamic noise in the raw single trial and the limitations of the Off-ROI subtraction technique, careful consideration must be taken when examining ArcLight single trials. Taken together, our results suggest that while there are aspects of the wide-field ArcLight imaging that reflect features of the LFP, the ArcLight fluorescence contains different and potentially additional information about cortical activation.

\subsection{ArcLight Excitation Causes Substantial Hemodynamic Noise in Recordings}

Hemodynamic noise is a known issue for in vivo imaging of fluorophores with blue-green excitation and emission ${ }^{19,42}$ due to the overlap with the absorption spectrum of hemoglobin. Most of the current GEVIs including ArcLight, Butterfly 1.2, and $\mathrm{mNeon}-\mathrm{Ace}^{8}$ all share blue-green excitation and emission wavelengths that cause tremendous noise for in vivo imaging. GEVIs based on FRET voltage probes, including Butterfly VSFP 2.1, have advantages in in vivo systems due to the ratiometric approach of the two fluorescence signals, which allows direct subtraction of a scaled hemodynamic signal. However, even these ratiometric approaches still require additional post hoc analysis to remove the properly scaled hemodynamic components from the recorded signals, which has been shown to be a nontrivial issue. ${ }^{19,64}$ For the non-FRET-based imaging methodology of ArcLight, we found that the ongoing hemodynamic noise required additional post hoc processing through Off-ROI subtraction to gain access to single-trial responses in the S1 cortex of the anesthetized mouse.

\subsection{Limitations of the Off-ROI Subtraction Method as a Tool for Removing Hemodynamic Noise}

Throughout this work, we implemented a scaled Off-ROI subtraction method to improve our ArcLight response and reduce ongoing hemodynamic noise, similar to techniques employed traditionally in processing in vivo wide-field imaging responses in voltage sensitive recording. ${ }^{33,43,62,65,66}$ However, this technique has several assumptions and limitations. By scaling and subtracting an Off-ROI region, we are making general assumptions about the shared dynamics of the noise spatially across the image. While this Off-ROI subtraction method does have success in removing temporal hemodynamic noise, careful considerations must be taken for spatial information, especially in locations far from the ROI. Additionally, the general assumption is that the observed signal of interest is spatially confined, and care should be taken for signals not confined to a particular brain region. We compared the mean of the raw data and the postprocessed ROI subtracted obtained similar spatial signals (Fig. 11). Additionally, similar to other reference methods, ${ }^{43,67}$ we are subtracting shared information, which may include spatiotemporal brain states, or evoked responses. To avoid these 
issues, we defined a 1-mm radius, which separates our ROI and the model template. This assumption is region specific to the S1 mouse barrel cortex, and therefore, must be adapted based on anatomy and functional responses. Overall, the methods described here detail the basis of a general model for subtracting common noise; however, there is a demand for better techniques for removing hemodynamic noise from spectrally overlapped excitation wavelengths. Furthermore, future development of brighter and more redshifted GEVIs will greatly reduce the hemodynamic influence in the recorded fluorescence signal.

\subsection{Comparison of ArcLight Cortical Responses to Previously Reported Voltage Sensors}

In this work, we focused on the performance of ArcLight to reflect the spatial and temporal evoked response in the $S 1$ barrel cortex. Although we did not directly compare ArcLight to other GEVIs, the whisker evoked fluorescence responses are within the range of the published temporal dynamics of voltage sensitive indicators in similar preparations. ${ }^{20,21,23}$ Very few studies have conducted cortical recordings using the same preparations described in this work; therefore, a direct comparison between GEVIs remains difficult. However, certain features of the mesoscopic whisker evoked S1 cortical response are consistent across imaging sensors and probes. We observed a strong sensory driven cortical response that produced fluorescence changes similar to other GEVIs (VSFP Butterfly 1.2 and VSFP 2.3) and VSDs (RH1691). These features include a sharp rising transient event lasting $\sim 30$ to $50 \mathrm{~ms},{ }^{20,21,23}$ and a longer tail that follows the response and decays over a period of a few hundreds of milliseconds. ${ }^{3,23}$ Additionally, the average evoked response SNR for ArcLight was similar to other GEVIs; however, in a single trial, the SNR was substantially worse which required the post hoc Off-ROI subtraction method to recover these signals. We observed that ArcLight was able to represent content only up to $\sim 20 \mathrm{~Hz}$ whisker stimulation; however, how much of this limitation is due to the imaging modality versus the dynamics of the pathway is unclear. Other FRET-based sensors have also reported frequencies of up to $20 \mathrm{~Hz}$ to sensory stimuli in other systems using similar techniques. ${ }^{19,68}$ Recently developed GEVIs including ASAP $1,{ }^{69}$ Ace-mNeon, ${ }^{6}$ Mac-mCitrine,${ }^{70}$ and Quasar1 report higher temporal resolution; however, most of these voltage probes have not yet been fully tested in vivo. We found ArcLight imaging had a slow rate of photobleaching with consistent responses recorded over a duration of $\sim 35 \mathrm{~min}$ of uninterrupted imaging (Fig. 3). Currently, there is no perfect GEVI that combines dynamic fluorescence range, photostability, large signals, and fast temporal dynamics. The results of this work suggest that ArcLight is capable of reporting sensory evoked responses in the cortex and can be used chronically to measure over many days to weeks.

\subsection{Future Applications of ArcLight and GEVI Imaging}

In summary, this work has demonstrated the potential use of the GEVI ArcLight as an in vivo method for investigating cortical circuits on a mesoscopic scale in the awake and anesthetized animal. GEVIs in general show tremendous promise in providing voltage measurements from small networks of cells simultaneously that can be selected based on genetic markers and have the potential to ultimately offer single cell resolution.
Like the many variants of opsins used, each study should select the proper GEVI for that particular work. ArcLight is a single example that provides a clear and reliable response to sensory stimuli in the sensory cortex, and we speculate would be ideal for behavioral experiments that require long imaging sessions. However, due to the potentially largely hemodynamic noise caused by the spectral overlap with hemoglobin, the user must take special precautions to remove this noise. Here, we utilized a scaled Off-ROI method that may not be applicable for all studies. Based on our results, ArcLight would be well-suited for in vivo experiments where a single fluorophore is desired, for example, during paired optogenetics or multispectral imaging of multiple cell types. Moreover, the true advantage of using a GEVI such as ArcLight is the ability to record the evoked response over the course of months and in the awake animal. Here, we found that both awake and repeated imaging experiments are feasible using the ArcLight voltage sensor. Future work is clearly needed to optimize the imaging and analytical techniques of these sensitive fluorescent probes.

\section{Appendix}

\section{A1 Removal of Hemodynamic Frequency using Notch Filtering}

Due to the hemodynamic noise found in the captured fluorescence signal (Fig. 3), our first approach for removing the noise was a simple common notch filter at the hemodynamic frequencies (main and corresponding first harmonic). We found that the hemodynamic signal we observed was correlated with the ongoing heart rate of the animal (Fig. 3) between $\sim 5$ and $10 \mathrm{~Hz}$, which corresponds to a heart rate between 300 and $600 \mathrm{bpm}$. This estimated heart rate range matches the typical physiological heart rate of an anesthetized mouse. Two examples (Fig. 9) are of ArcLight evoked sensory responses and the corresponding power spectral density of the evoked fluorescence with and without notch filtering (second-order Butterworth, bandstop between 5 and $20 \mathrm{~Hz}$ ). We found that due to the total event time of the ArcLight response $(\sim 100$ to $300 \mathrm{~ms}$ ), simple notch filtering at 5 to $20 \mathrm{~Hz}$ would greatly disrupt the ArcLight waveform.

\section{A2 Removal of Hemodynamic Frequency Using Off-ROI Subtraction Methods}

Throughout this work, we utilized an Off-ROI to subtract ongoing hemodynamic noise observed in the fluorescence signal. This Off-ROI technique utilized the highly correlated structure of the hemodynamic signal through the cortical tissue to subtract common noise. Two figures detail the removal of the hemodynamic noise through the Off-ROI subtraction (Fig. 10) and the effect of the Off-ROI placement on the spatiotemporal response (Fig. 11).

\section{A3 Noninjected ArcLight Control}

To confirm that any effects of autofluorescence do not significantly affect our results, we conducted additional experiments examining a noninjected animal to determine the potential influence of the intrinsic autofluorescence on the spatial extent of the response. First, we mapped the S1 barrel cortex using 
(a) Animal ID- PB_17

comparison of mean temporal response

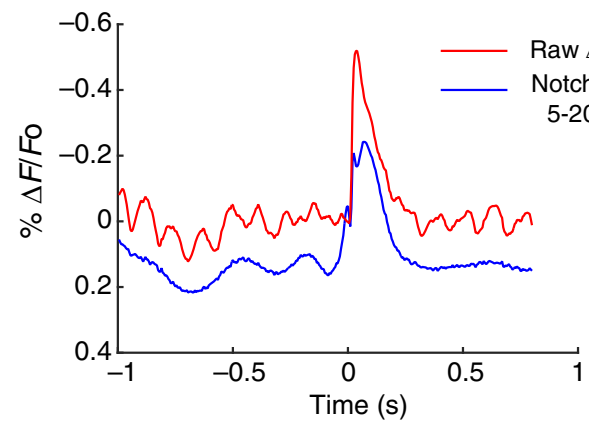

Comparison of frequency spectra

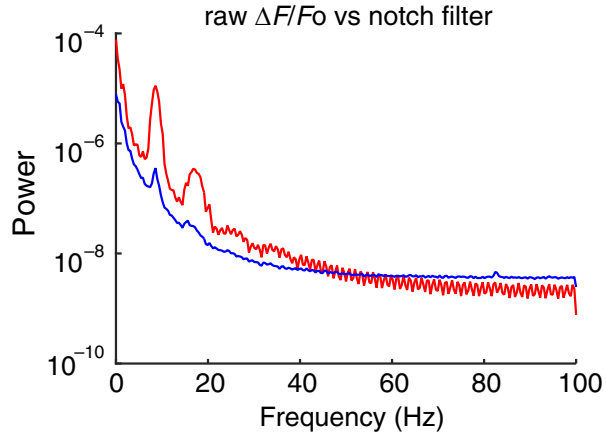

(b) Animal ID- PB_18

comparison of mean temporal response

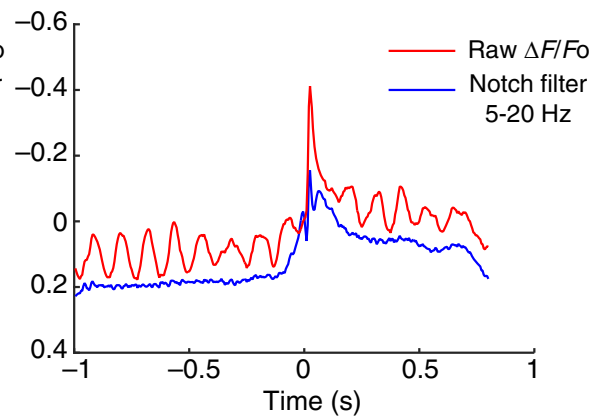

Comparison of frequency spectra raw $\Delta F / F O$ vs notch filter

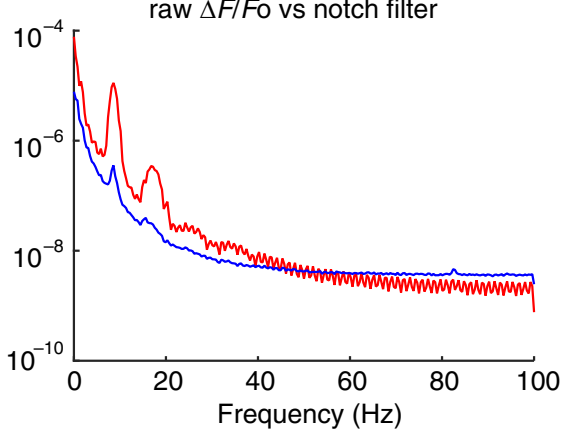

Fig. 9 Notch filtering of fluorescence response. (a, top) Average (102 trials) ROI time series ArcLight fluorescence response during a whisker deflection (stimulus delivered at time $=0$ ). The red trace response shows a strong fluorescence response to the sensory stimulus. The blue trace illustrates the change in waveform caused by notch filtering. (a, bottom) Corresponding average power spectral density of the fluorescence signal. Notice the large peaks at $\sim 10$ and $20 \mathrm{~Hz}$ corresponding to the hemodynamic response. (b) Same as (a) but for a different experiment.

(a)

Animal PSD of raw vs subtracted fluorescence $(n=1,102$ trials)

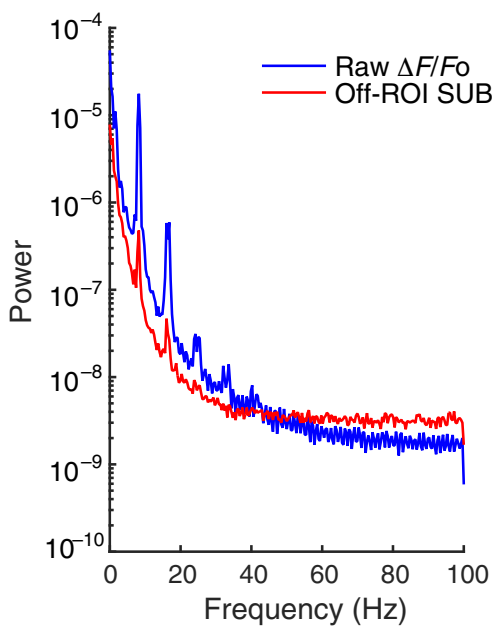

(b)

Average PSD of raw vs subtracted fluorescence $(n=23)$

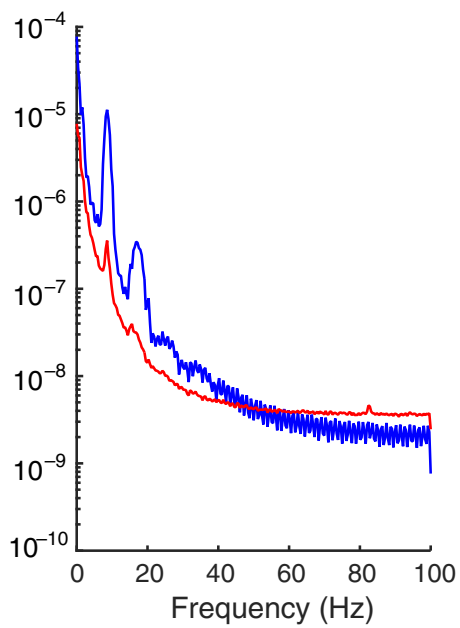

(c)

Change in $7-10 \mathrm{~Hz}$ power $n=23$

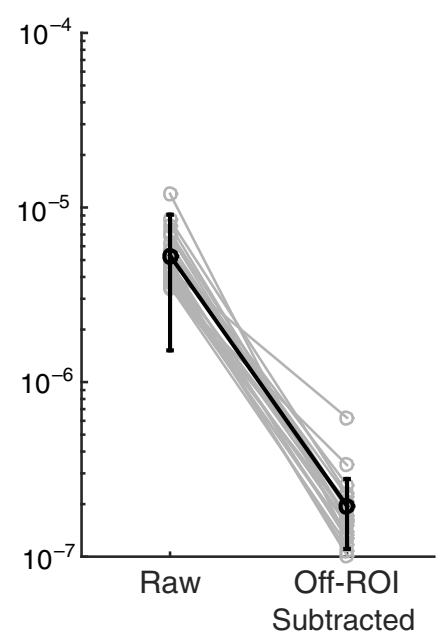

Fig. 10 Reduction of hemodynamic noise. (a) Average power spectra of the ROI of the fluorescence before (blue-raw $\% \Delta F / F_{0}$ ) and after (red $\% \Delta F / F_{0}$ ROI sub.) Off-ROI subtraction. The Off-ROI subtraction reduces the peak of 7 to $10 \mathrm{~Hz}$ frequency power. (b) Similar to (a), the average power spectra of the $\mathrm{ROI}$ across experiments $(n=23)$ before (blue) and after (red) Off-ROI subtraction. (c) Off-ROI subtraction causes a mean reduction of $95.8 \%( \pm 2.8 \% \mathrm{SD}, n=23, p=0.0033$, paired $t$-test) frequency power in the 7 to 10 band. 
(a)
Mean response
(102 trials)
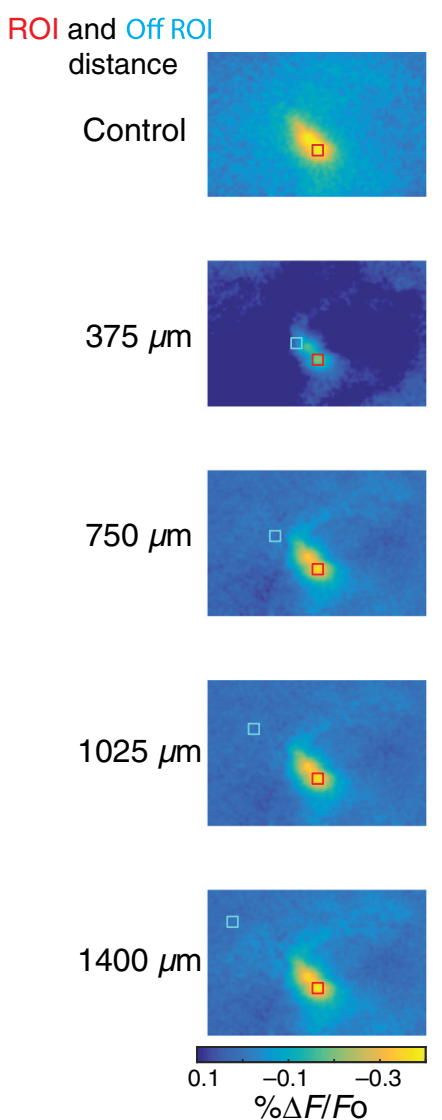

(b)
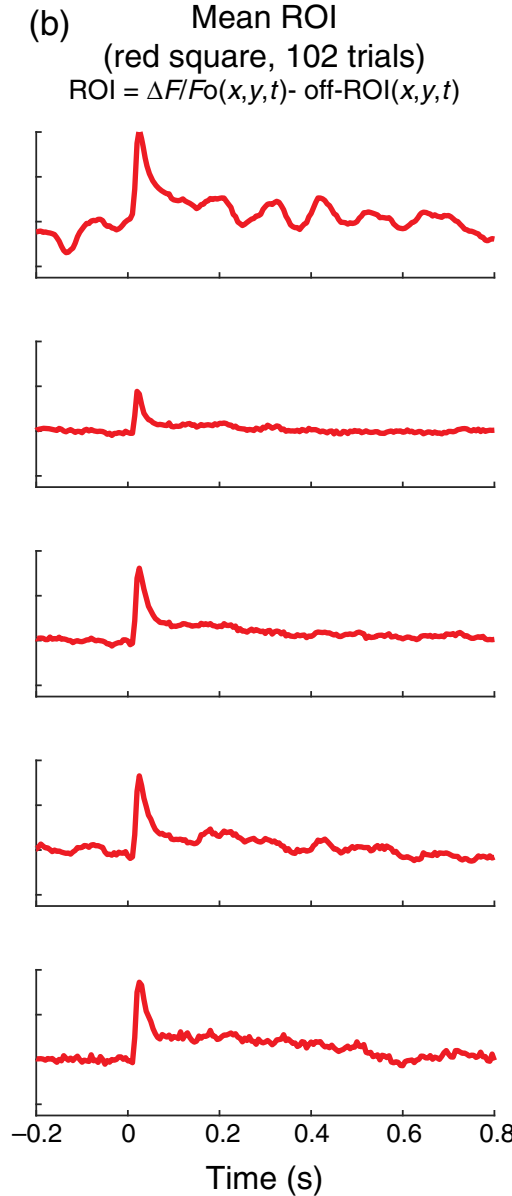

(c)

Mean off-ROI

(blue square, 102 trials)
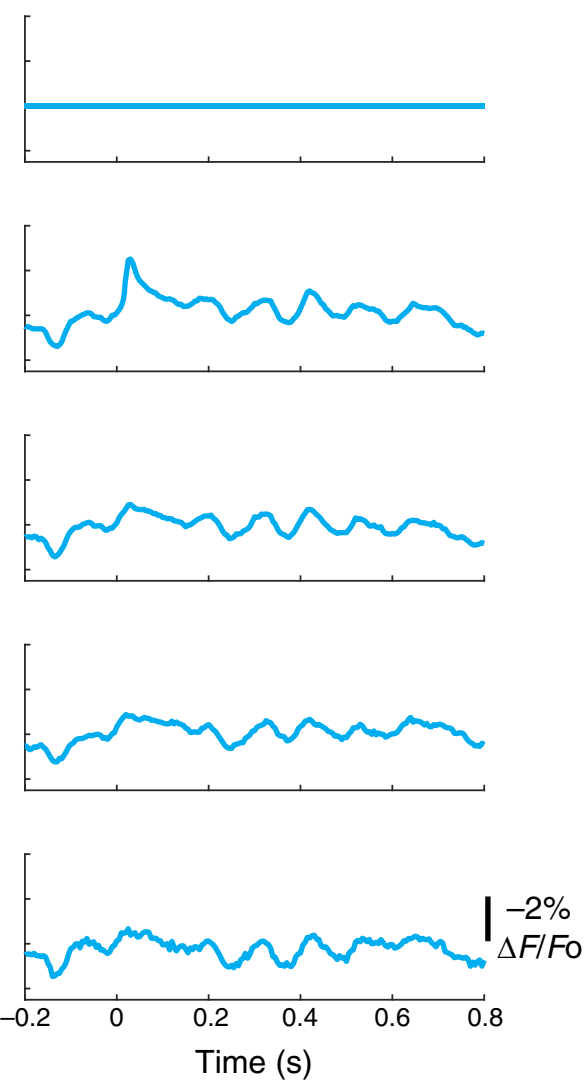

Fig. 11 Effect of the position of the Off-ROI on the evoked spatial and temporal response. (a, top) Off-ROI subtracted mean spatial response $25 \mathrm{~ms}$ after stimulus (102 trials). Red square highlights the location of the ROI. Blue square highlights the Off-ROI to be scaled and subtracted. (b, top) The average temporal trace taken from the spatial image ROI (red square) after Off-ROI subtraction. (c, top) The average temporal trace taken from the spatial image Off-ROI (blue square) to be subtracted from the ROI. Each row shows the same plots for increasing distance between the Off-ROI and the ROI (375 $\mu \mathrm{m}$ per row). As the Off-ROI becomes more spatially separated from the $\mathrm{ROI}$, there is a reduction in the influence on the spatial and temporal trace. After $\sim 600 \mu \mathrm{m}$, the Off-ROI does not interfere with the evoked response.

intrinsic imaging (see Sec. 2.4) to determine the proper location of the corresponding whisker sensitive cortical region. Due to the slow temporal dynamics and overall small change in signal, we measured the intrinsic response to a repetitive stimulus over several seconds. Figure 12(a) shows a temporal average (across 20 trials) of the intrinsic response in a time window of 1 to $2 \mathrm{~s}$ after the onset of the strong repetitive whisker stimulation (1500 deg / s at $10 \mathrm{~Hz}$ for $5 \mathrm{~s}$ ).

After we mapped the region using the intrinsic signal, we set up the system for ArcLight imaging described in detail in this paper and applied the same single whisker punctate stimuli used in a majority of the study [single $1200 \mathrm{deg} / \mathrm{s}$ sawtooth ( $\tau=8 \mathrm{~ms}$ ) stimulus]. In Fig. 12(b), we compared the intrinsically identified whisker region [Fig. 12(a)] to responses using the ArcLight setup and experimental parameters used throughout this work. Specifically, we sampled the same area at $200 \mathrm{~Hz}$ with blue excitation $(465 \mathrm{~nm})$ along with the excitation and emission filters as described in Sec. 2. Figure 12(b, left) shows the averaged spatial response over the 700-ms window poststimulus (100 trials), with no apparent qualitative difference between the identified ROI (red) and other Off-ROIs (blue and aqua). The 700-ms window corresponds to the approximate length of the average S1 cortical response used throughout this paper [Fig. 2(c)]. Figure 12(b, right) shows the time series of integrated activity within these ROIs. We found no quantitative difference between the evoked response in the identified (red) ROI and the background, prestimulus activity, indicating that there was no appreciable evoked response. [E1: mean prestimulus fluorescence $(-700$ to $0 \mathrm{~ms}) \% \Delta F / F_{0}-0.0073 \pm$ $0.0057 \mathrm{SD}$, mean poststimulus fluorescence (0 to $700 \mathrm{~ms}$ ) $\% \Delta F / F_{0}, 0.0063 \pm 0.059 \mathrm{SD}, p=0.102$, paired $t$-test].

In the noninjected mouse, in the ArcLight setup [Fig. 12(b)], we also observe oscillatory responses $(8$ to $10 \mathrm{~Hz}$ ) across both whiskers similar to the observed hemodynamic signal in the single-trial responses, shown in Fig. 3. These hemodynamic oscillations can still be seen in the $\mathrm{C} 2$ and E1 trial average in Fig. 12(b). These results further suggest that this oscillatory signal is not representative of ongoing membrane potential fluctuations but is due to the blood flow across the cortical surface. Based on these controls, we expect that the observed stimulus 
(a) Mean intrinsic response $625 \mathrm{~nm} @ 10 \mathrm{~Hz}$

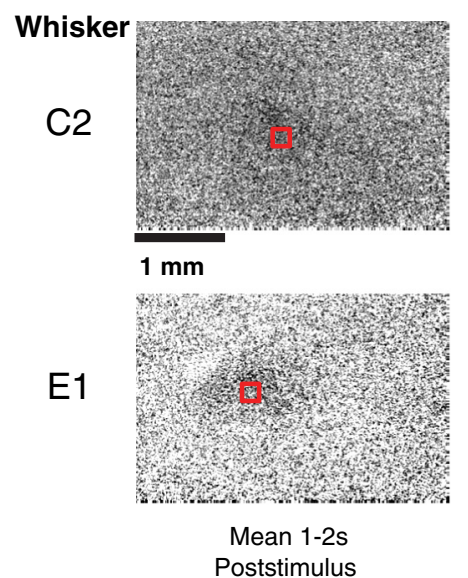

(b) ArcLight setup (figure 1A) 465 nm w/filters @ 200Hz
Mean temporal response (100 trials)

Whisker stimulus
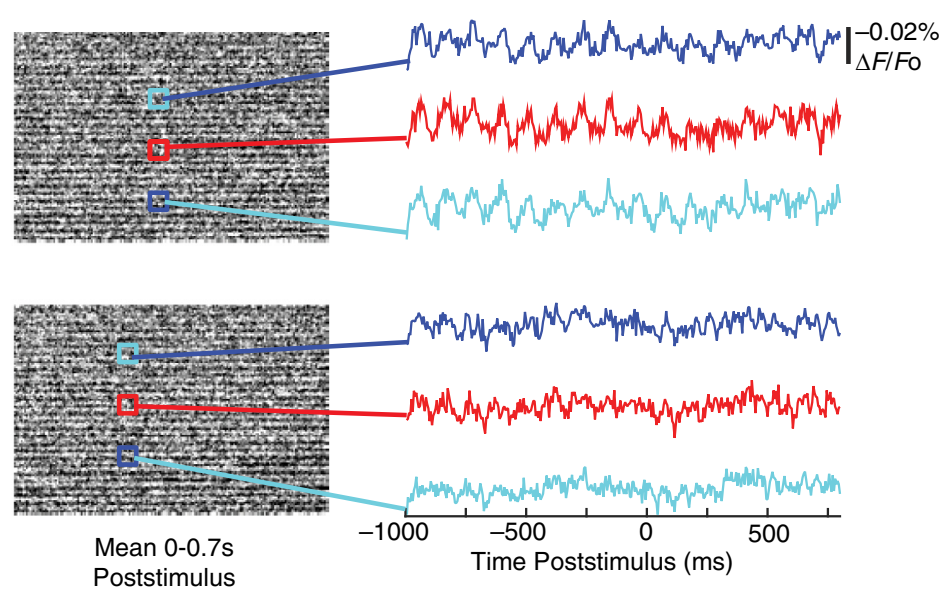

Fig. 12 Noninjected control of intrinsic response during whisker stimulus. (a) Mean intrinsic mapped response (20 trials). The primary cortical barrel was first identified using the intrinsic response. The cortical surface was thinned and prepared as described in detail in the Methods section. A Thorlabs red $(625 \mathrm{~nm})$ LED illuminated the skull during repetitive stimulation of a single whisker (top whisker C2, bottom E1). The thumbnails show the temporal average between 1 and $2 s$ after the onset of the repetitive stimulus. The spatial response was normalized and subtracted by the average background response, to increase the contrast of the evoked signal, and smoothed with a 200- $\mu$ m Gaussian filter. (b) Single whisker deflection with the ArcLight setup. Each thumbnail (left) represents the mean response (100 trials) 0 to $0.7 \mathrm{~s}$ poststimulus to the same whisker shown in (a). The imaging setup was switched from the intrinsic imaging configuration (a) to the ArcLight configuration [Fig. 1(a)], sampling at $200 \mathrm{~Hz}$. The signals have been analyzed using the same methods (however, no Off-ROI subtraction was used). Temporal traces of the integrated fluorescence in each of the illustrated ROls are shown (right). Each ROI (blue, red, aqua boxes) corresponds to the temporal traces shown in the right. The timing of the whisker deflection is shown at the top.

evoked fluorescence response shown throughout our study is most likely due to the changes in neural activity associated with ArcLight and not due to autofluorescence.

\section{Disclosures}

The authors declare no competing financial interests.

\section{Acknowledgments}

This paper was supported by the National Institute of Neurological Disorders and Stroke (NINDS): Award Nos. R01NS48285 (PI: G.B. Stanley), R01NS085447 (PI: G. B. Stanley), U01NS094302 (PI: D. Jaeger, and G.B. Stanley), and NS09869 (PI: P.Y. Borden); National Institute of Mental Health (NIMH): Award No. U01MH106027 (PI: G.B. Stanley, C.R. Forest). The authors would like to thank William Stoy, Ilya Kolb, and Timothy Lee, as well as other members of the Precision Biosystems Lab at Georgia Tech, for additional support and advice on imaging. Additionally, we thank Kyle Blum for feedback on imaging analysis and illustrations. We would also like to acknowledge He Zheng for pilot work on the ArcLight imaging. Furthermore, we would like to thank Vincent A. Pieribone for supplying us with the initial ArcLight protein and for imaging advice.

\section{References}

1. L. Jin et al., "Single action potentials and subthreshold electrical events imaged in neurons with a fluorescent protein voltage probe," Neuron 75(5), 779-785 (2012).

2. G. Cao et al., "Genetically targeted optical electrophysiology in intact neural circuits," Cell 154(4), 904-913 (2013).
3. W. Akemann et al., "Imaging brain electric signals with genetically targeted voltage-sensitive fluorescent proteins," Nat. Methods 7(8), 643-649 (2010)

4. W. Akemann et al., "Imaging neural circuit dynamics with a voltagesensitive fluorescent protein," J. Neurophysiol. 108(8), 2323-2337 (2012).

5. D. R. Hochbaum et al., "All-optical electrophysiology in mammalian neurons using engineered microbial rhodopsins," Nat. Methods 11(8), 825-833 (2014).

6. Y. Gong et al., "High-speed recording of neural spikes in awake mice and flies with a fluorescent voltage sensor," Science 350(6266), 13611366 (2015)

7. R. Nakajima et al., "Optogenetic monitoring of synaptic activity with genetically encoded voltage indicators," Front. Synaptic Neurosci. 8, 1-9 (2016).

8. D. Storace et al., "Toward better genetically encoded sensors of membrane potential," Trends Neurosci. 39(5), 277-289 (2016).

9. T. Knöpfel, Y. Gallero-Salas, and C. Song, "Genetically encoded voltage indicators for large scale cortical imaging come of age," Curr. Opin. Chem. Biol. 27, 75-83 (2015).

10. M. Z. Lin and M. J. Schnitzer, "Genetically encoded indicators of neuronal activity," Nat. Neurosci. 19(9), 1142-1153 (2016).

11. S. D. Antic, R. M. Empson, and T. Knopfel, "Voltage imaging to understand connections and functions of neuronal circuits," J. Neurophysiol. 116, 135-152 (2016).

12. Z. Han et al., "Mechanistic studies of the genetically encoded fluorescent protein voltage probe ArcLight," PLoS One 9(11), e113873 (2014).

13. D. Brinks, A. J. Klein, and A. E. Cohen, "Two-photon lifetime imaging of voltage indicating proteins as a probe of absolute membrane voltage," Biophys. J. 109(5), 914-921 (2015).

14. D. Sitaraman et al., "Propagation of homeostatic sleep signals by segregated synaptic microcircuits of the Drosophila mushroom body," Curr. Biol. 25(22), 2915-2927 (2015).

15. D. Raccuglia et al., "Presynaptic GABA receptors mediate temporal contrast enhancement in Drosophila olfactory sensory neurons and modulate odor-driven behavioral kinetics," eNeuro 3(4) (2016). 
16. M. Klein et al., "Sensory determinants of behavioral dynamics in Drosophila thermotaxis," Proc. Natl. Acad. Sci. 112(2), E220-E229 (2015).

17. N. C. Klapoetke et al., "Independent optical excitation of distinct neural populations," Nat. Methods 11(3), 338-346 (2014).

18. D. A. Storace et al., "Monitoring brain activity with protein voltage and calcium sensors," Sci. Rep. 5, 10212 (2015).

19. M. Carandini et al., "Imaging the awake visual cortex with a genetically encoded voltage indicator," J. Neurosci. 35(1), 53-63 (2015).

20. E. F. Civillico and D. Contreras, "Integration of evoked responses in supragranular cortex studied with optical recordings in vivo," J. Neurophysiol. 96(1), 336-351 (2006).

21. I. Ferezou, S. Bolea, and C. C. H. Petersen, "Visualizing the cortical representation of whisker touch: voltage-sensitive dye imaging in freely moving mice," Neuron 50(4), 617-629 (2006).

22. I. Ferezou et al., "Spatiotemporal dynamics of cortical sensorimotor integration in behaving mice," Neuron 56(5), 907-923 (2007).

23. H. Mutoh et al., "Comparative performance of a genetically-encoded voltage indicator and a blue voltage sensitive dye for large scale cortical voltage imaging," Front. Cell. Neurosci. 9, 147 (2015).

24. Q. Wang, R. M. Webber, and G. B. Stanley, "Thalamic synchrony and the adaptive gating of information flow to cortex," Nat. Neurosci. 13(12), 1534-1541 (2010).

25. N. M. Razali and Y. B. Wah, "Power comparisons of ShapiroWilk, Kolmogorov-Smirnov, Lilliefors and Anderson-Darling tests," J. Stat. Model. Anal. 2(1), 21-33 (2011).

26. H. Lütcke et al., "Optical recording of neuronal activity with a genetically-encoded calcium indicator in anesthetized and freely moving mice," Front. Neural Circuits 4, 9 (2010).

27. Z. Han et al., "Fluorescent protein voltage probes derived from ArcLight that respond to membrane voltage changes with fast kinetics," PLoS One 8(11), e81295 (2013).

28. C. A. Gollnick et al., "Response reliability observed with voltage-sensitive dye imaging of cortical layer 2/3: the probability of activation hypothesis," J. Neurophysiol. 115(5), 2456-2469 (2016).

29. M. C. Stüttgen and C. Schwarz, "Psychophysical and neurometric detection performance under stimulus uncertainty," Nat. Neurosci. 11(9), 1091-1099 (2008).

30. M. Shoykhet, D. Doherty, and D. J. Simons, "Coding of deflection velocity and amplitude by whisker primary afferent neurons: implications for higher level processing," Somatosens. Mot. Res. 17(2), 171180 (2009).

31. J. Wolfe et al., "Texture coding in the rat whisker system: slip-stick versus differential resonance," PLoS Biol. 6(8), e215 (2008).

32. C. C. H. Petersen, A. Grinvald, and B. Sakmann, "Spatiotemporal dynamics of sensory responses in layer $2 / 3$ of rat barrel cortex measured in vivo by voltage-sensitive dye imaging combined with wholecell voltage recordings and neuron reconstructions," J. Neurosci. 23(4), 1298-1309 (2003).

33. M. T. Lippert et al., "Methods for voltage-sensitive dye imaging of rat cortical activity with high signal-to-noise ratio," J. Neurophysiol. 98(1), 502-512 (2007).

34. Q. Wang et al., "Erratum: voltage-sensitive dye imaging reveals improved topographic activation of cortex in response to manipulation of thalamic microstimulation parameters," J. Neural Eng. 9(5), 59601 (2012).

35. D. C. Millard, Q. Wang, and G. B. Stanley, "Nonlinear system identification of the thalamocortical circuit in response to thalamic microstimulation," in 2011 5th Int. IEEE/EMBS Conf. Neural Engineering (NER 2011) (2011).

36. E. Welker and H. Van der Loos, "Quantitative correlation between barrel-field size and the sensory innervation of the whiskerpad: a comparative study in six strains of mice bred for different patterns of mystacial vibrissae," J. Neurosci. 6(11), 3355-3373 (1986).

37. T. A. Woolsey and J. R. Wann, "Areal changes in mouse cortical barrels following vibrissal damage at different postnatal ages," J. Comp. Neurol. 170(1), 53-66 (1976).

38. A. Ramirez et al., "Spatiotemporal receptive fields of barrel cortex revealed by reverse correlation of synaptic input," Nat. Neurosci. 17(6), 866-875 (2014).

39. E. E. Kwegyir-Afful, H. T. Kyriazi, and D. J. Simons, "Weaker feedforward inhibition accounts for less pronounced thalamocortical response transformation in mouse vs. rat barrels," J. Neurophysiol. 110(10), 2378-2392 (2013).

40. D. Kleinfeld and K. R. Delaney, "Distributed representation of vibrissa movement in the upper layers of somatosensory cortex revealed with voltage-sensitive dyes," J. Comp. Neurol. 375(1), 89-108 (1996).

41. H. Jia et al., "In vivo two-photon imaging of sensory-evoked dendritic calcium signals in cortical neurons," Nat. Protoc. 6(1), 28-35 (2010).

42. E. M. C. Hillman, "Optical brain imaging in vivo: techniques and applications from animal to man," J. Biomed. Opt. 12(5), 051402 (2007).

43. H. Raguet et al., "Spatially structured sparse morphological component separation for voltage-sensitive dye optical imaging," J. Neurosci. Methods 257, 76-96 (2016).

44. S. I. Al-Juboori et al., "Light scattering properties vary across different regions of the adult mouse brain," PLoS One 8(7), e67626 (2013).

45. G. Buzsáki, C. A. Anastassiou, and C. Koch, "The origin of extracellular fields and currents-EEG, ECoG, LFP and spikes," Nat. Rev. Neurosci. 13(6), 407-420 (2012).

46. B. Haider et al., "Millisecond coupling of local field potentials to synaptic currents in the awake visual cortex," Neuron 90(1), 35-42 (2016).

47. M. Okun, A. Naim, and I. Lampl, "The subthreshold relation between cortical local field potential and neuronal firing unveiled by intracellular recordings in awake rats," J. Neurosci. 30(12), 4440-4448 (2010).

48. M. Okun, "Artefactual origin of biphasic cortical spike-LFP correlation," J. Comput. Neurosci. 42(1), 31-35 (2017).

49. V. Khatri, R. M. Bruno, and D. J. Simons, "Stimulus-specific and stimulus-nonspecific firing synchrony and its modulation by sensory adaptation in the whisker-to-barrel pathway," J. Neurophysiol. 101(5), 2328-2338 (2009).

50. V. Khatri, "Adaptation in thalamic barreloid and cortical barrel neurons to periodic whisker deflections varying in frequency and velocity," J. Neurophysiol. 92(6), 3244-3254 (2004).

51. R. M. Webber and G. B. Stanley, "Nonlinear encoding of tactile patterns in the barrel cortex," J. Neurophysiol. 91(5), 2010-2022 (2004).

52. A. Boloori, R. A. Jenks, and G. B. Stanley, "Encoding and decoding cortical representations of tactile features in the vibrissa system," J. Neurosci. 30, 9990-10005 (2010).

53. H. T. Kyriazi, G. E. Carvell, and D. J. Simons, "OFF response transformations in the whisker/barrel system," J. Neurophysiol. 72(1), 392401 (1994).

54. E. E. Kwegyir-Afful et al., "The role of thalamic inputs in surround receptive fields of barrel neurons," J. Neurosci. 25(25), 5926-5934 (2005).

55. J. Anderson et al., "Stimulus dependence of two-state fluctuations of membrane potential in cat visual cortex," Nat. Neurosci. 3(6), 617621 (2000).

56. D. S. Reich et al., "Response variability and timing precision of neuronal spike trains in vivo," J. Neurophysiol. 77(5), 2836-2841 (1997).

57. R. D. Singla, J. Wang, and D. K. Singla, "Regulation of notch 1 signaling in THP-1 cells enhances $\mathrm{M}_{2}$ macrophage differentiation," $\mathrm{Am}$. $J$. Physiol. Heart Circ. Physiol. 307(11), H1634 (2014).

58. B. R. Kallman, H. Kim, and K. Scott, "Excitation and inhibition onto central courtship neurons biases Drosophila mate choice," Elife 4, e11188 (2015).

59. P. R. Haynes, B. L. Christmann, and L. C. Griffith, "A single pair of neurons links sleep to memory consolidation in Drosophila melanogaster," Elife 4, e03868 (2015).

60. E. Shtoyerman et al., "Long-term optical imaging and spectroscopy reveal mechanisms underlying the intrinsic signal and stability of cortical maps in V1 of behaving monkeys," J. Neurosci. 20(21), 8111-8121 (2000).

61. E. Seidemann et al., "Calcium imaging with genetically encoded indicators in behaving primates," Elife 5, e16178 (2016).

62. T. H. Grandy, S. A. Greenfield, and I. M. Devonshire, "An evaluation of in vivo voltage-sensitive dyes: pharmacological side effects and signalto-noise ratios after effective removal of brain-pulsation artifacts," J. Neurophysiol. 108, 2931-2945 (2012).

63. V. Reyes-Puerta et al., "Propagation of spontaneous slow-wave activity across columns and layers of the adult rat barrel cortex in vivo," Brain Struct. Funct. 221(9), 4429-4449 (2016).

64. B. A. Wilt et al., "Photon shot noise limits on optical detection of neuronal spikes and estimation of spike timing," Biophys. J. 104, 51-62 (2013). 
65. A. Arieli et al., "Dynamics of ongoing activity: explanation of the large variability in evoked cortical responses," Science 273(5283), 18681871 (1996).

66. A. Grinvald et al., "Cortical point-spread function and long-range lateral interactions revealed by real-time optical imaging of macaque monkey primary visual cortex," J. Neurosci. 14, 2545-2568 (1994).

67. K. A. Ludwig et al., "Chronic neural recordings using silicon microelectrode arrays electrochemically deposited with a poly(3, 4-ethylenedioxythiophene) (PEDOT) film,” J. Neural Eng. 3(1), 59-70 (2006).

68. L. Madisen et al., "Transgenic mice for intersectional targeting of neural sensors and effectors with high specificity and performance," Neuron 85(5), 942-958 (2015).

69. F. St-Pierre et al., "High-fidelity optical reporting of neuronal electrical activity with an ultrafast fluorescent voltage sensor," Nat. Neurosci. 17(6), 884-889 (2014).

70. Y. Gong et al., "Imaging neural spiking in brain tissue using FRETopsin protein voltage sensors," Nat. Commun. 5, 3674 (2014).

Peter Y. Borden received his BSE degree in bioengineering from Rice University in 2008. He is currently a doctoral student at Georgia Institute of Technology and Emory University in the joint Biomedical Engineering Graduate Program. His current research aims to understand thalamocortical processing related to sensory processing.

Alex D. Ortiz received his bachelor of science degree in biochemistry from Tulane University in 2006 and his master of business administration from Georgia Institute of Technology in 2016. He has been a research associate in the Department of Biomedical Engineering at Georgia Institute of Technology since 2009.

Christian Waiblinger received his $\mathrm{PhD}$ in neuroscience from Werner Reichardt Centre for Integrative Neuroscience Systems Neurophysiology in 2015 . He is currently a postdoctoral fellow at the Georgia Institute of Technology. His research focuses on understanding cortical and thalamic sensory processing during behavior.
Audrey J. Sederberg received her $\mathrm{PhD}$ in physics from Princeton University in 2011. She is currently a postdoctoral fellow at the Georgia Institute of Technology. Her research focuses on understanding the role of cortical state on sensory processing.

Arthur E. Morrissette received his BS degree in biomedical engineering from Georgia Institute of Technology in 2013. Since then, he has been a doctoral student at Emory University in the Neuroscience Graduate Program. His current research aims to understand cortical and basal ganglia processing related to sensorimotor control.

Craig R. Forest is an associate professor of mechanical engineering at Georgia Tech, where he also holds program faculty positions in bioengineering and biomedical engineering. He was a fellow at the Allen Brain Institute, Seattle, Washington, USA, and he is one of the inaugural recipients of the NIH BRAIN Initiative Grants, a national research effort to invent the next generation of neuroscience and neuroengineering tools.

Dieter Jaeger's research combines cellular and systems electrophysiological recordings from rodents with detailed computer modeling to examine how neurons in the basal ganglia and in the cerebellum process their inputs. Of particular interest is the function of inhibition and of active neural properties in network processing. The ultimate goal is to understand the motor function of basal ganglia and cerebellar networks during behavior in normal and disease states.

Garrett B. Stanley received his PhD from the University of California, Berkeley, in mechanical engineering with postdoctoral work in neuroscience in the Department of Molecular and Cellular Biology at Berkeley. He is the Carol Ann and David D. Flanagan professor in the Department of Biomedical Engineering at the Georgia Institute of Technology and Emory University. The work of his laboratory focuses on information processing in sensory pathways, with an emphasis on the thalamocortical circuit. 